
218074621

\title{
Substrate-dependent cluster density dynamics in bacterial phosphotransferase system permeases
}

Gustavo Benevides Martins, Giacomo Giacomelli and Marc Bramkamp*

Ludwig-Maximilians-Universität München, Fakultät Biologie, Großhaderner Straße 2-

4, 82152 Planegg-Martinsried, Germany

Running title: Spatial dynamics of bacterial PTS permeases

Keywords: Protein dynamics, PTS, membrane occupancy, fluorescence microscopy, localization microscopy, protein cluster density

${ }^{*}$ Corresponding author: Marc Bramkamp 


\section{Abstract}

Bacteria take up carbohydrates by membrane-integral sugar specific phosphoenolpyruvate-dependent carbohydrate:phosphotransferase systems (PTS). Although PTS is at the heart of bacterial carbon uptake and centrally involved in regulation of carbon metabolism, little is known about localization and putative oligomerization of the permease subunits (EII) of PTS. Here, we analyzed localization of the fructose specific PtsF and the glucose specific PtsG transporters from $C$. glutamicum using widefield and single molecule localization microscopy. PtsG and PtsF form membrane embedded clusters that localize in a punctate pattern within the cell membrane. The size, number and fluorescence of the observed clusters changes upon presence or absence of the transported substrate. In presence of the transport substrate clusters significantly increased in size. Photo-activated localization microscopy (PALM) data revealed that, in presence of different carbon sources, the number of Ell protein events per cluster remain the same, however the density of PTS molecules within a cluster reduces. Our work reveals a simple mechanism for efficient membrane occupancy regulation. Clusters of PTS Ell transporters are densely packed in absence of a suitable substrate. In presence of a transport substrate the Ell proteins in individual clusters occupy larger membrane areas, thereby decreasing protein density in individual clusters. This mechanism allows for efficient use of the limited membrane space under varying growth conditions without need of protein removal and re-synthesis.

\section{Importance}


42 The carbohydrate transport system PTS is centrally involved in the regulation of

43 sugar metabolism. Although much is known about the regulatory interaction, the 44 genetic control and the structure/function relationship of the individual PTS 45 components, we know almost nothing about the spatio-temporal organization of the PTS proteins within the cell. We find dynamic clustering of PTS permeases in

47 Corynebacterium glutamicum. Using single molecule resolution photo-activated 48 localization microscopy we could show that PTS Ell protein cluster are dynamically changing protein density upon substrate availability. Our findings imply a novel strategy of regulating limited membrane space efficiently. Furthermore, these data will provide important insights in modelling carbohydrate fluxes in cells, since current models assume a homogeneous distribution of PTS permeases within the membrane. 


\section{Introduction}

In heterotrophic bacteria, uptake of suitable carbohydrates is an essential task for the cells in their quest for food and, hence, subject to meticulous regulation. In growth media with several carbon sources, many bacteria including enteric bacteria like Escherichia coli, use preferred sugars such as glucose first. This leads to the wellknown diauxic growth behavior $(1,2)$. Regulation of sequential carbohydrate usage and transport of carbohydrates is often governed by an enzyme complex termed phosphoenolpyruvate-dependent carbohydrate:phosphotransferase systems (PTS) (3-8). Interestingly, some bacteria are known to co-ferment different carbohydrates with Corynebacterim glutamicum being a prominent example $(9,10)$. C. glutamicum is a facultative anaerobic, chemoheterotroph that is used in the large scale industrial production of amino acids (11-13). In industrial applications, the main feedstock used in most of the established fermentation processes are molasses and starch hydrolysates, which contain a broad spectrum of simple carbohydrates, but mostly glucose, fructose and sucrose. These sugars are taken up and phosphorylated during transport into the cell via PTS. This means that worldwide amino acid production using this organism depends heavily on the PTS transport activities (14). The PTS consists of two common energy-coupling cytoplasmic proteins, enzyme I (EI) and histidine protein (HPr), which are encoded by pts/ (cg2117) and ptsH (cg2121), respectively, and a series of sugar-specific enzyme II (EII) complexes located in the membrane. The Ell complexes are typically divided into three protein domains, EIIA, EIIB and EIIC, whose organization differs between bacteria, ranging from organisms in which the three Ell domains are fused in a single protein to a variety of differently fused and unfused domains $(3,7)$. In E. coli, the glucose PTS 
subunit EllA is detached, while in B. subtilis and C. glutamicum, all three subunits are fused together. In enteric bacteria EIIA (also termed EIIA ${ }^{c r r}$, since the coding gene is abbreviated $\mathrm{crr}$ ), is at the heart of a complex regulatory system that governs the quest for food (4-6). EllA ${ }^{\text {crr }}$ interacts with several transport proteins, such as the lactose permease LacY, thereby inhibiting their activity. This leads to the inducer exclusion effect (15). The accumulation of phosphorylated EllA ${ }^{\text {crr }}$, a sign of starvation, activates the adenylate cyclase, leading to cyclic AMP (cAMP) production. The second messenger cAMP acts as coactivator for the Crp protein cumulating in permanent catabolite repression in enteric bacteria. The passage of specific sugars through the membrane is catalyzed by the transmembrane EIIC part of the system (16). Structural analysis of EIIC subunits revealed that they likely work as dimers. They contain a substrate recognition and a transport part (17). The PTS couple transport to the conversion of carbohydrates into their respective phosphoesters using the energy of phosphoryl group translocation. The phosphoryl group from phosphoenolpyruvate (PEP) is transferred to EI, HPr, EIIA, EIIB and finally to the substrate as it is transported across the membrane. In C. glutamicum four PTS transport system have been described. The organization of the C. glutamicum PTS for fructose, glucose and sucrose are represented in figure 1. ptsG (cg1537) encodes the glucose-specific membrane integral EIIBCA transporter, while ptsS (cg2925) encodes the sucrose-specific ElIBCA, and ptsF (cg2120) the fructose-specific EIIABC. A fourth PTS belongs to the L-ascorbate-type family and was characterized by R. Hvorup, et al. (18)(not depicted in the graphic).

Ell expression is known to be induced by the presence of its transported sugar (19), and regulation of PTS gene expression is mainly controlled at the stage of transcription initiation or at transcription elongation in C. glutamicum (19). The 
mechanism for control of PTS gene expression differs for the respective PTS genes. The transcriptional regulator SugR, encoded by sugR (cg2115) is a deoxyribonucleoside repressor (DeoR)-type regulator and is located in the fructosePTS operon. It has been shown to repress not only pts $F, p t s G$ and ptsS expression in the absence of the transported sugar, but also the general PTS genes pts/ and ptsH in C. glutamicum (20-22). Post-transcriptional regulation of PtsG in E. coli is governed by SgrS, a small Hfq-binding RNA, that is induced by phosphor-sugar accumulation in the cytosol. It forms a ribonucleo-protein complex with Hfq and RNaseE, resulting in translational repression and degradation of ptsG mRNA (23, 24).

The catabolite repressor/activator FruR (also known as Cra), encoded by fruR down regulates the fructose-PTS operon in E. coli, Salmonella typhimurium and Pseudomonas putida when the sugar is not available $(22,25,26)$. In P. putida, FruB acts by binding as a dimer to a single palindromic sequence within the $c r a / f r u B$ intergenic region (which is different to those of E. coli and S. typhimurium), and that only fructose-1-phosphate can lift such down regulation (26). In S. pneumonia, exogenous auto-inducer 2 (Al-2) induces fruA and fruB, which encode PtsF and 1phosphofructokinase in that organism (27). Expression of enzyme I and HPr increases in the presence of different PTS sugars. In Streptomyces coelicolor and Lactobacillus casei, glucose is reported to be the most effective inducing sugar of pts/ and ptsH expression $(28,29)$. In C. glutamicum, the role of FruR (cg2118) is distinct from the one observed in other bacteria. It represses the transcription of the fruRptkB1-pts $F$ operon, as well as $p t s /$ and $p t s H$ in presence of fructose, decreasing the induction effect of fructose and adjusting the expression level of pts genes to prevent overflow of PTS sugars (30) 
129 It has been proposed that the PTS can be described as a central "cellular functioning unit" [CFU], regulating the cells quest for food (4-6). However, to fully describe CFUs, precise knowledge about spatio-temporal localization of its components is required. There is surprisingly little knowledge on subcellular localization of PTS components. Early immune-gold labelling with the E. coli Ell ${ }^{\mathrm{mtl}}$ showed membrane localization that can be interpreted as patchy with no preferred subcellular enrichment (31). A more recent study showed that the $E$. coli BglF (the $\beta$-glucoside specific Ell permease) is located throughout the plasma membrane (32). However, deconvolved images and plasmid born expression do not allow for unambiguous differentiation between a uniform and a patchy localization. The general components of the E. coli PTS, El and $\mathrm{HPr}$ in contrast localize to the cell poles $(32,33)$. HPr localization changes in presence of the transport substrate from polar to dispersed throughout the cytoplasm.

Here, we describe the localization of two specific Ell components in C. glutamicum. We have constructed translational fusions of $p t s G$ and $p t s F$ encoding PtsG (El|glc) and PtsF (Ellfrc) with different fluorescent proteins. Constructs were designed as allelic replacements, ensuring native genetic control and all constructs were shown to be fully functional. Widefield fluorescence microscopy revealed that Ell complexes localize as dynamic clusters in the cell membrane. PtsG and PtsF Ell components exclude each other within the membrane compartment, but PtsF co-localize with components of the respiratory chain, ruling out a specific membrane domain for carbohydrate transport only. Importantly, we observed an increase in PTS Ell cluster size when the specific sugar substrate was present. This increase in cluster sized coincided with a decrease in cluster number. Using photo-activated single molecule fluorescence microscopy (PALM) we were able to quantitatively address PTS 
153

154

155

156

157

158

159

160

161

162

163

164

165

166

167

168

169

170

171

172

173

174

175

dynamics. PALM data clearly show that PTS Ell cluster are covering a larger membrane area when their transport substrate is presence. Importantly, under these conditions the complexes do not contain more Ell molecules, but rather reduce protein density within clusters. Thus, actively transporting PTS permeases are spreading apart and non-transporting complexes are densely packed. This dynamic arrangement of the PTS offers a simple mechanism for efficient membrane occupancy under varying nutrient conditions.

\section{Results}

\section{Construction of functional PTS fusion proteins}

To investigate subcellular localization and membrane occupancy of PTS Ell permeases, we constructed C. glutamicum strains with fluorescent fusions to PtsF and PtsG, resulting in strains CGM001 and CGM002 expressing mCherry-PtsF and mNeonGreen-PtsG, respectively (Table S2). We have not included the sucrose specific PTS since sucrose is a disaccharide composed of fructose and glucose and we wanted to first test for the specific influence of glucose and fructose. $C$. glutamicum is importing sucrose via the sucrose specific PTS. The phosphorylated sucrose is cleaved intracellularly and the resulting fructose molecule is exported and taken up by the fructose specific PtsF. Hence, a clean separation between sucrose and fructose effects is not easily possible. To check for potential colocalization of the glucose and fructose specific Ell permeases a double labelled strain was constructed. To this end, CGM001 was used as a background for incorporation of mNeonGreen-linker-ptsG via allelic replacement, resulting in the double-labelled 
strain ptsF::mCherry-ptsF, ptsG::mNeonGreen-linker-ptsG (CGM003) (Fig. 2A). In order to gain quantitative single molecule resolution data of PTS localization and clustering behavior, strains containing PtsF (CGM004) and PtsG (CGM005) tagged with photoactivatable mCherry (PAmCherry) were constructed for PALM microscopy. To avoid overexpression and artificial expression heterogeneity, the fusion constructs were all inserted as allelic replacement in the genome of wild type C. glutamicum (RES 167) cells. To test functionality of the constructed fusion proteins, growth experiments and sugar consumption assays via HPLC were performed. Strains CGM001, expressing mCherry-PtsF (Fig. 2B), CGM002 expressing mNeonGreenPtsG (Fig. 2C) and the double labelled strain CGM003 displayed wild type like growth behavior (Figs. 2D and 2E). The growth rates of wild type did not differ from the constructed strains and ranged from 0.28 to $0.29 \mathrm{~h}^{-1}$ (Tab. 1), showing that the tagged PTS systems were functioning wild type-like. We also measured sugar consumption rates in CGXII supplemented with $2 \%$ fructose or $2 \%$ glucose. The specific sugar consumption rates $q_{s}$ were highly similar for wild type and mutant strains (Tab. 1). The measured qs values for the wild type of around $0.19 \mathrm{~g} \mathrm{~g}^{-1} \mathrm{~h}^{-1}$ for glucose and $0.13 \mathrm{~g} \mathrm{~g}^{-1} \mathrm{~h}^{-1}$ for fructose are very well within the expected range for the observed growth rate (34). The strain carrying the fluorescently labelled PtsG had consumption rates of $0.18 \mathrm{~g} \mathrm{~g}^{-1} \mathrm{~h}^{-1}$ for glucose and the strain with the PtsF fusion construct displayed consumption rates of $0.14 \mathrm{~g} \mathrm{~g}^{-1} \mathrm{~h}^{-1}$ for fructose. In summary we conclude that the constructed fusions are fully functional. It should be noted here, that in a process to gain functional translational fusions, several constructs were made that were all not further analyzed when they turned out to be non-functional.

Protein localization studies using translational fusions can be hampered by protein degradation and subsequent imaging of free fluorophore. Therefore, we investigated 
protein degradation by western blotting with anti-mCherry for mCherry and PAmCherry fusions, and in-gel fluorescence for mNeonGreen fusions. For each strain, it was possible to identify one the band corresponding to their full length fusion protein, or oligomers, revealing that no major degradation was present (Fig. S1). We therefore conclude that localization studies with these strains should reveal the native localization of the full length PTS Ell permeases.

\section{C. glutamicum Ell frc Ellglc spatial dynamics upon presence or absence of the} transported sugars

We first wanted to investigate whether PTS Ell proteins distribute uniformly or in clusters along the cytoplasmic membrane under aerobic conditions in presence of the transported sugars. To this end, fluorescence microscopy was performed on C. glutamicum strains expressing mNeonGreen-PtsG and mCherry-PtsF grown in CGXII supplemented with the transported sugars as sole carbon sources. Analysis of fluorescence images showed that both proteins form membrane embedded clusters that localize punctually within the cell membrane with no preferred positions (Fig. 3AB). Ellfru and El|glc foci of varying intensity were observed, suggesting complexes with different amounts of proteins. Most cells contained few bright and intense foci that were randomly distributed. However, bright foci seemed to be present with higher frequency close to cell poles (Fig. 3A-B).

We next wanted to test whether cell shape and the surface/volume ratio might have an influence on the PTS behavior. Therefore, we analyzed PTS localization in L-form bacteria, generated out of the corresponding strains. Cells expressing mNeonGreenPtsG and mCherry-PtsF were grown in MXM/CGXII medium supplemented with the 
225 transported sugars in presence of D-cycloserine (DCS). DCS is a cyclic analogue of D-alanine, acting against alanine racemase (Alr) and D-alanine:D-alanine ligase

227 (Ddl), two crucial enzymes in the cytosolic stages of peptidoglycan synthesis, bypassing the need to block cell wall synthesis genetically. As DCS was previously used in Mycobacterium tuberculosis (35), which shares the characteristic cell wall common to all Corynebacterineae, it was chosen for L-form formation with $C$. glutamicum. L-form bacteria require an osmotically stabilized medium. Usually, the osmoprotective environment would be achieved by adding sucrose to the media, but since this sugar is taken up by the sucrose specific PtsS, we complemented the Lform medium with xylose, a carbohydrate that cannot be efficiently metabolized by $C$. glutamicum. Fluorescence microscopy with L-forms revealed the same PtsF and PtsG clustering that was observed in rod-shaped cells (Fig. 3C-D). We conclude from this, that cluster formation of PTS Ell permeases may be an intrinsic property of these enzymes and not depend on cell shape or surface/volume ratios.

We next wanted to investigate whether presence or absence of the specific PTS carbohydrates influence the PTS Ell complexes localization and expression. To this end, the strains containing fluorescent reporters linked to the Ell complexes were grown in CGXII minimal medium with $2 \%$ glucose, fructose or acetate as sole carbon sources to early exponential phase, and fluorescent microscopy was subsequently performed. Importantly, both proteins are expressed in the absence of their specific transported sugars (Fig. $4 \mathrm{~A}-\mathrm{C}$ ) and there were no differences among early, mid and late log phases (data not shown). Observed PtsG/F foci did not show evident colocalization (Fig. 4D), suggesting that phosphotransferase systems localize independently within the membrane. This is an important observation, because the 
control we analyzed localization of the ElI fru complexes with a protein from the respiratory chain. Here, we have chosen the succinate dehydrogenase subunit $A$ (SdhA) in combination with PTS Ell. We did observe a large degree of co-localization between PtsF and SdhA (Fig. 4E). These data rule out that in C. glutamicum components of the respiratory chain and the PTS would occupy specific and different membrane domains.

After the observation that clustering and expression of PTS Ell occurs even in absence of the transported substrate, we next wanted to determine the influence of different carbon sources on PTS foci number per cell, foci area, fluorescence, and how much of the membrane space is covered by PTS. Analysis of fluorescent images of ptsF::mCherry-ptsF, ptsG::mNeonGreen-Linker-ptsG both in rod shaped cells and L-forms grown in minimal medium under different carbon sources revealed that the number of PtsF/G clusters per cell was significantly decreased in presence of the transported sugar in rod-shaped cells (Fig. 5 and Tab. 2). Statistically, the number of PtsF clusters per cell was equal in acetate and glucose treatments, and differed in rod-shaped and L-form cells grown in fructose. Regarding PtsG foci number per cell in rod-shaped, cells grown in fructose or acetate did not differ statistically, whilst when grown in glucose, the values were significantly lower. L-form cells showed a tendency to have more PTS foci, having higher averages (3.3 per cell for both PtsF/G), being statistically different from all the other treatments. The absence of a cell wall in L-forms results in significantly larger cells (mean cell area values: rodshaped $3.6 \mu \mathrm{m}^{2}$, L-forms $8.9 \mu \mathrm{m}^{2}$ ), which leads to a drastic reduction in the cell surface/volume (S/V) ratio. Despite being grown in medium containing the same carbon source, the observed increase in number of PtsF/G clusters in L-forms 
suggests that PTS Ell foci pattern can be changed in response to variations in cell area, volume or morphology.

Previous studies have revealed that the expression of the C. glutamicum PTS is induced when cells are cultured in presence of the transported sugar $(19-21,36)$. In order to test the influence of different carbon sources on PTS foci, PtsF/G Corrected Total Fluorescence (CTF) was calculated with ImageJ based on the obtained fluorescence microscopy images and corrected for the cell area (Fig. 6A,B). Analysis of CTF of tagged proteins served as a proxy to assess levels of protein expression and concentration, and both PtsF and PtsG were similar regarding the fluorescence readings in this work. Although all the treatments were statistically different from each other, CTF of both proteins increased in presence of the transported substrate and decreased in its absence. This indicates that indeed, PtsF and PtsG expression was induced in presence of the transported substrate. When compared to rod shaped cells grown in the same carbon source, the CTF of L-forms was over 4 times higher (4,5x for PtsF and 4,1x for PtsG), this suggests that the number of PTS Ell complexes within the cell membrane increases with the cell area and number of chromosomes, since large L-forms are known to larger numbers of chromosomes (37).

The areas of the PtsF/G foci of cells grown in different carbon sources are summarized in Figure 7A and Table 3. In rod-shaped cells, PtsF foci area values were statistically similar when cells were grown in glucose or acetate, and significantly higher in fructose. Likewise, PtsG foci area in glucose was 3 times higher than in fructose, and 4 times than in acetate. However, unlike PtsF, the PtsG cluster area values obtained for cells grown in fructose were higher than cells grown in 
acetate. L-Form cells exhibited the highest PtsF/G foci areas compared to every other condition in rod-shaped cells, suggesting that a larger membrane area create more space to be occupied by transmembrane proteins.

In order to know how much of the cytoplasmic membrane is occupied by PTS, the ratio foci area/cell area (Fig. 7B) was calculated. Rod-shaped cells had the highest ratios when in presence of the transported sugar, meaning that more membrane space is reallocated for PTS proteins in such conditions. In average, PtsG ratio in presence of glucose was 3 times higher than in fructose, and 4 times than in acetate. Although less dramatic, the increase in PtsF ratio while in presence of fructose was still significant: 1,8 times higher than in glucose, and 1,45 higher than acetate. In general, L-forms exhibited larger PTS clusters, in a higher overall number, and fluorescence. This leads to the logical assumption that a larger cell area increases the area available for protein insertion, possibly resulting in more transmembrane PTS proteins. However, the foci area/cell area ratio of L-Forms was not higher than other rod-shaped cells. In fact, despite PtsF L-form values being higher than in rodshaped cells grown in glucose or acetate, they were lower than rod-shaped cells in fructose (Table 4). Roughly, the same was observed for PtsG: even though L-forms had a higher foci area/cell area ratio than rod-shaped cells in general, there was no statistical difference between their values and rod-shaped grown in fructose, a condition where PtsG is hardly induced. 
321 The data obtained with widefield microscopy suggested that PTS Ell cluster

322 rearrange when their specific transport substrate is present. However,

323 epifluorescence microscopy is limited by the diffraction limit, and a series of

324 fundamental information about PTS Ell clusters such as density or number of

325 molecules cannot be obtained. Therefore, the next step we took towards a deeper

326 and more quantitative understanding of PTS Ell complex dynamics was to use single

327 molecule photo-activated localization microscopy (PALM) data. To this end, the

328 construction of strains tagged with photoactivatable mCherry (PAmCherry)

329 ptsG::PAmCherry-Linker-ptsG and ptsF::PAmCherry-ptsF was carried out the same

330 way as the previous strains and was also shown to be fully functional as judged by

331 sugar uptake and growth rates (Fig. S2). The observed clustering pattern in

epifluorescence was confirmed by PALM data of cells expressing PAmCherry-PtsG

333 (strain CGM005) (Fig. 8 A,B). Also, PAmCherry-PtsF formed membrane embedded

clusters, but the low number of events detected per cell made it impossible to obtain

significant statistical analysis that would show cluster density changes (Fig. S3).

However, we observed a tendency that is in accord with the data obtained for PtsG.

337 For analysis of PALM data we first needed to precisely define what a valid cluster is.

Clusters are defined as regions of high density separated by regions of lower density, and the distribution our data suggests is that there are three populations separated

$X \geq 25$ events (Fig. S4). The first population could in theory still be composed of PTS complexes close to each other by mere coincidence. Although this randomness effect composed of $\geq 10$ and $\geq 25$ events were analyzed regarding their maximum span, 
exponential distribution of PTS cluster size is reminiscent to that observed with chemotaxis receptors (38) and therefore suggests a stochastic self-assembly process. We detected around $177.65(\mathrm{sd}=92.48)$ PTS Ellglc events per $\mu \mathrm{m}^{2}$ cell area in glucose, $111.22(s d=49.51)$ events per $\mu m^{2}$ in fructose, and $114.69(s d=60.28)$ events per $\mu \mathrm{m}^{2}$ in acetate.

The increase in foci area observed in epifluorescence brought up the question whether the cluster area increases due to an increase in number of PTS complexes present in each cluster, or to a rearrangement of the same number of Ell permeases per cluster. At single molecule resolution, foci area can be estimated by the cluster maximum span (Fig. $8 \mathrm{C}$ ), which is the maximum distance between two events belonging to the same cluster. PAmCherry-PtsG clusters from populations B and C exhibited significantly higher max span under the presence of glucose when compared to fructose or acetate, which exhibited no statistical difference among each other, corroborating our findings with epifluorescence, where in presence of the transported sugars, PtsG/F covered a larger membrane area. The analysis of these same populations of clusters revealed that cells in presence of glucose exhibited significantly higher number of PtsG clusters per $\mu \mathrm{m}^{2}$ when compared to fructose and acetate, which exhibited no differences among each other (Fig. 8D). CTF readings of epifluorescence images showed increased values when cells expressing mCherryPtsF and mNeonGreen-PtsG were grown in presence of the transported sugars, suggesting increased expression of PTS complexes under these conditions. This data correlates with our PALM data for PamCherry-PtsG (Fig. 9A). In presence of glucose, a significantly higher number of events per $\mu \mathrm{m}^{2}$ was observed, meaning that the number of Ellglc in the cytoplasmic membrane increases with the addition of glucose in the medium, again confirming induction of $p t s G$ in presence of glucose as 
expected. Our epifluorescence data suggested that in presence of the transported sugar, PtsF/G assemble in larger complexes, while the overall number of clusters per cell decreases. However, our PALM analysis of cells expressing PAmCherry-PtsG in glucose showed an increase in the number of clusters and events per $\mu \mathrm{m}^{2}$. Single molecule detection allowed visualization of clusters with fewer proteins, thereby explaining the apparent discrepancy to the widefield microscopy data. While in widefield the number of bright, visible foci decreased (while their area increased) when the transport substrate was present, in PALM we observed more clusters with lower protein numbers that likely account from substrate induced gene expression.

Although the CTF of cells increases in presence of glucose, PALM data showed that the number of PAmCherry-PtsG events per cluster remain the same independently of the carbon source, suggesting that the carbon source does not affect the number of PTS proteins present in each cluster (Fig. 9B). This is an important finding since larger cluster size could have been the trivial consequence of more protein in the cell. Since we have found that the number of PTS Ell molecules in a cluster remained similar in presence or absence of the transported substrate, we wanted to analyze the protein density in individual clusters. The local density is defined as the number of events present in a squared area of side $50 \mathrm{~nm}$ centered on the event. The average density of events is defined as the arithmetic average of local density of the events composing the cluster. PAmCherry-PtsG clusters of population C exhibited significant lower average density values in presence of glucose (Fig. 9C), meaning that Elglc complexes belonging to the same cluster localize further apart from each other when in presence of glucose, thereby occupying a larger membrane area. 


\section{Discussion}

Most bacteria transport various carbohydrates via a PTS system. The tremendous advantage of using a PTS coupled transport is that the substrate is phosphorylated in the process of import (hence the term group translocation), thereby effectively being removed from the chemical equilibrium of outside and inside concentrations. This allows the highly effective transport even under low external carbohydrate concentrations. Yet, a second, similarly important feature of PTS transport is that the complex PTS is composed of a sensory part and a regulatory part $(4,6)$. In enteric bacteria the ElIA ${ }^{\text {crr }}$ protein is a central component in the complex regulation cascade and a main part in the observed catabolite repression. ElIA ${ }^{\text {crr }}$ binds and inhibits the lactose permease as well as the GlpK protein in E. coli, while the phophsphorylated P EIIA ${ }^{\text {crr }}$ activates the adenylate cycles (3). It is therefore not surprising that the EIIA component in $E$. coli is therefore a soluble protein that can dissociate from the membrane bound EIIBC complex. In line with this function subcellular localization of EllA ${ }^{\text {Crr }}$ was shown to be dispersed in the cytoplasm (32). Also the general PTS components $\mathrm{HPr}$ is involved in regulation in $E$. coli and phosphorylates the transcriptional regulator BglG. For $\mathrm{HPr}$ a polar localization was described in E. coli that is alleviated when transport substrates are present (32). These data were in line with early suggestions that the PTS complex should act in multi-protein complexes (39), thereby improving its function (40). A major unsolved question in our understanding is still the unclear localization and assembly of the membrane embedded permease part of the PTS. Up to date there is only one report about the localization of an Ell complex, the BgIF in E. coli (32). However, BgIF localization studies were performed with a plasmid borne expression system, potentially causing 
overexpression and therefore masking the possible clustering behavior of this PTS protein. While the general setup of proteins making a functional PTS is conserved in most bacteria, their genetic arrangement and regulatory role differs greatly among bacteria. In the high GC, gram positive C. glutamicum, four specific PTS were described (14). C. glutamicum differs greatly from organisms in which the PTS is well studied by the fact that it prefers utilization of several carbon sources simultaneously $(9,10)$. Hence, for most carbon sources $C$. C. glutamicum does not show diauxic growth behavior (except under conditions with ethanol or glutamate plus glucose). It is therefore not surprising that the permease subunits are fusion proteins composed of EIIABC and that they do not have a diffusible EllA subunit. Our data using an Nterminal fluorescent fusion for the fructose specific EIIABC confirms clear membrane localization of the entire complex. It was generally assumed that the Ell parts of the PTS are uniformly distributed in the cytoplasmic membrane, similar to other transport proteins, such as the Hxt hexose transporter in yeast (41), and the mentioned BglF (32). However, data on subcellular localization of transport proteins is rather scarce. This may in part be based on the relatively low copy number of many transport proteins that renders microscopy localization studies difficult. We have succeeded here in the subcellular localization of the EIIC permease parts of the fructose and glucose specific PTS. Both membrane integral transporters show a clustered membrane distribution. Importantly, both fusion constructs are fully functional based on their growth rates and the respective carbon sources consumption rates. Since the constructs replaced the native allele, we confidently assume also a wild type like copy number. Wide field microscopy not only revealed the heterogeneous, clustered localization of the two PTS components, but also showed that they hardly co-localize. 
444 PtsF does co-localize with the succinate dehydrogenase, a protein of the TCA cycle and the respiratory chain. This finding indicates that in $C$. glutamicum proteins of the respiratory chain and transport proteins can co-occur in the same membrane region. The membrane economy model proposed by Zhuang et al. (42) suggests that bacteria regulate their membrane composition based on efficient usage of the limited membrane space and idealized a model in which a clear membrane separation of proteins involved in transport and respiration might occupy distinct membrane areas. For C. glutamicum we can exclude such a strict spatial distribution.

C. glutamicum PTS Ell expression is known to be induced by the presence of the transported sugars (19), and regulation of the PTS gene expression is mainly controlled at the stage of transcription initiation or at transcription elongation (19). Our epifluorescence data support the induction of PtsF and PtsG in presence of the transported sugars by the increase in total fluorescence readings of cells under these conditions. Moreover, PtsF/G clusters increase in both size and foci area/cell area ratio, occupying more membrane space upon presence of glucose or fructose, while the overall number of large complexes, visible in widefield microscopy, decreases. signaling and scaffold proteins. Prime examples of membrane receptor clustering are

462 the chemotactic receptors. A seminal publication describing the self-organization of 463 the E. coli chemotaxis receptors using localization microscopy reveled that polar 464 chemotaxis clusters mature by a stochastic assembly of smaller clusters and single receptor proteins (38). Other membrane proteins, such as flotillins $(43,44)$, the OXPHOS components Nuo, CydAB, CyoABCD, SdhABC $(45,46)$ (and own results, 467 see Fig, 4E) have also been shown to localize in clusters of various size in the 
membrane. It is reasonable to assume that the clustering in these cases is based on stochastic self-assembly and that cluster formation is important for function. In contrast to scaffolding proteins such as flotillins $(44,47)$, it is not immediately apparent why a transport protein should cluster for an improved function. The PTS system however, is, as we described above, not only a transport system, but also a signalling device. Hence, clustering may be advantageous for signalling. In this context, it is interesting to note that a direct link between the PTS and the chemotaxis system has been described in E. coli $(48,49)$.

Here, we could show with single molecule localization microscopy, that the observed PTS cluster dynamically change their cluster density based on presence or absence of substrate. PAmCherry-PtsG PALM data showed that the presence of glucose in the medium induces expression and leads to cells exhibiting a higher number of larger clusters composed of $10<x<25$ and $25<x$ events. These clusters showed a higher Cluster Max Span, but lower Average Density of Clusters values, meaning that the number of PtsG Ell proteins around another decreases in presence of the transported sugar. At the same time, the distribution of number of events per cluster remained the same independently of the carbon source. These data strongly suggest a spatial rearrangement of PTS complexes, which might be a strategy to increase efficiency of membrane space utilization, or a form of regulation employed by cells (Fig. 10). Furthermore, a similarity between the function of chemotaxis receptors and the membrane bound Ell complexes of the PTS is the crucial involvement of phosphorylation reactions. For the chemotaxis receptor it has been described that clustering improves phosphorylation reactions. Strikingly, it has also been described that different chemoreceptor densities lead to different kinase activity based on the local concentration of the receptors (50). In widefield microscopy these clusters are 
493

494

495

496

497

498

499

500

501

502

503

504

505

506

507

508

509

510

511

512

513

514

515

polar localized, but when cells treated with an excess of attractant, polar clusters disperse and proteins distribute over a large area of the membrane. It has been proposed that signal amplification under conditions of high attractant concentration is not needed (51). In a similar concept one might speculate that the PTS Ell complexes in C. glutamicum increase their cluster density in absence or at very low concentration of the transport substrate. Under such conditions a positive cooperative effect might be useful. At higher concentrations of the transport substrate this cooperativity may not be advantageous and hence the PTS spreads over a larger membrane area. We observed a change in cluster size with PtsF and PtsG. However, statistically solid data were only achieved for PtsG due to the higher natural expression level. However, our widefield microscopy data further support this observation. This is the first time that a dynamic change in cluster density has been observed in cells and reveals an unexpected spatial regulation of the PTS. Several groups report data that suggest dimer formation of Ell permeases $(17,52,53)$. The nature of our experimental design in single molecule localization does not allow to distinguish between monomer and dimer in the membrane of $C$. glutamicum reliably.

Using artificial, induced L-form states, we could show that PTS cluster formation is not due to the rod-shape of the cells and the limited membrane surface. Although Lform cells show a grossly altered number of PTS clusters (likely owed to the higher expression as a result of the presence of multiple chromosomes), and also cluster size was larger, the same foci area/cell area ratio was retained, supporting the notion that membrane space is indeed a constraint that limits membrane occupancy by PTS proteins. However, clustering is an intrinsic function of the PTS Ell. 
516 Understanding the precise membrane coverage and spatio-temporal distribution of

517 the PTS systems will help to model and scale up growth specific sugar uptake in

518 industrial processes such as amino acid production in C. glutamicum. A kinetic model for glucose PTS in $E$. coli has been constructed $(54,55)$ and converted spatio-

520 temporally to address the possible effects of diffusion in the PTS systems. Based on

521 the model, it was concluded that the soluble E. coli ElIAglc remains with up to $20 \%$

522 close to the membrane $(55,56)$. Thus in larger cells such as eukaryotic cells (or as

523 shown here with L-forms) diffusion may disrupt efficient signaling due to diffusion

524 limitation and subsequent gradient formation. However, these data were made under

525 the assumption that membrane complexes as well as general PTS proteins are

526 uniformly distributed either in the membrane (permeases) or cytosol (general PTS components). In the light of the data provided in this work, kinetic flux models might be adapted.

Currently, it remains unclear what triggers this remarkable change in cluster conformation. Future works need to discriminate whether substrate binding or the transport (e.g. phosphorylation) of the carbohydrates is required. We will address this question by placing mutations within the phosphotransfer reaction site of EIIAB or by using carbohydrate mimetics that bind EIIAB, but do not get transported. Furthermore, it remains to be tested, whether the observed dynamic cluster response to substrates is a peculiar finding in C. glutamicum or whether this could be a general mechanism found in other bacteria such as firmicutes or enteric bacteria. 


\section{Bacterial strains and cloning}

541 Oligonucleotides, strains and plasmids used in this study are listed in Tables S1 and

542 S2, respectively. DNA manipulations and E. coli DH5a transformations were carried out using standard cloning methods (57), and all constructed plasmids were verified by DNA sequencing.

\section{Media and growth conditions}

For genetic manipulations, E. coli strain $\mathrm{DH} 5 a$ was grown at $37^{\circ} \mathrm{C}$ in Lysogeny Broth

(LB) medium supplemented, when appropriate, with kanamycin $25 \mu \mathrm{g} / \mathrm{ml}$. all microscopy and growth analysis. Acetate was used as a base carbon source, as it allows relatively good growth among non-PTS carbon sources in C. glutamicum. For all the experiments, strains were cultivated during the day in $5 \mathrm{ml}$ of LB medium and then diluted in $10 \mathrm{ml}$ of LB/CGXII medium and grown overnight. The following day the cultures were diluted to an optical density (OD) of 1.0 in $10 \mathrm{ml} \mathrm{CGXII.} \mathrm{For}$

557 microscopy, samples were taken in early exponential phase, when the OD reached 2.

558 Bacterial L-forms were grown in osmoprotective medium composed of $2 \times \mathrm{MXM}$ medium, pH 7 (40 mM MgCl $2,1 \mathrm{M}$ xylose, and $40 \mathrm{mM}$ maleic acid), mixed 1:1 with 2x 
562

563

564

565

566

567

568

569

570

571

572

573

574

575

576

577

578

579

580

581

582

583

sugar was used for osmotic protection instead of sucrose to avoid possible influences in the studied PTS complexes.

\section{Carbon consumption assays}

The quantification of glucose, fructose and acetate in the supernatants of cultures was performed by high-performance liquid chromatography (HPLC) using a Dionex UltiMate 3000 (Thermo Scientific) HPLC system equipped with an Aminex HPX-87H column (300 by $7.8 \mathrm{~mm}$; Bio-Rad). Isocratic elution was performed with $6 \mathrm{mM}$ $\mathrm{H}_{2} \mathrm{SO}_{4}$ at $80^{\circ} \mathrm{C}$ for $20 \mathrm{~min}$ at a flow rate of $0.6 \mathrm{ml} / \mathrm{min}$. Fructose and glucose were detected with a PDA-100 Photodiode Array Detector (Dionex) at $190 \mathrm{~nm}$, and acetate was detected with a PDA-100 Photodiode Array Detector (Dionex) at $206 \mathrm{~nm}$. Quantification was performed using calibration curves obtained with external standards.

\section{Fluorescence microscopy}

For standard fluorescence microscopy, cells were grown to early exponential phase as described before (59) and $1 \mu \mathrm{l}$ of culture was spread on a $1 \%$ agarose gel pad. The setup used for fluorescent microscopy consisted of a Delta Vision Elite (GE Healthcare, Applied Precision) equipped with an Insight SSI ${ }^{\mathrm{TM}}$ illumination, an X4 laser module and a Cool Snap HQ2 CCD camera was used (100x oil PSF U-Plan SApo1.4 NA objective). Digital image analysis, was performed with Fiji (60). The corrected total fluorescence (CTF) was calculated according to following formula: 
584 CTF $=$ Integrated Density - (Area of selected cell X Mean fluorescence of unspecific background readings) (61).

586

587

588

All statistical analysis and plotting were performed in RStudio and final image preparation was done in Adobe Photoshop CS2 (Adobe Systems Incorporated). All imaging experiments were performed several times with biological replicates, and PtsF/G foci analysis was performed in $>200$ cells.

\section{Photoactivated Localization Microscopy (PALM)}

After growing to early exponential phase in CGXII supplemented with the desired carbon source as described before, cultures were fixed through incubation for 30 minutes at $30^{\circ} \mathrm{C}$ in formaldehyde in a final concentration of $1 \% \mathrm{v} / \mathrm{v}$. Cells were then harvested by centrifugation and washed 3 times with PBS $+10 \mathrm{mM}$ glycin, to be finally resuspended to 2 ODunits (OD.mL ) in $200 \mu \mathrm{L}$ TSEMS (50 mM Tris, pH 7.4, 50 mM NaCl, 10 mM EDTA, 0.5 M sucrose, 1x Protease Inhibitor Cocktail (Sigma), 13.2 $\left.\mathrm{ml} \mathrm{H}_{2} \mathrm{O}\right)$.

Imaging was performed using a Zeiss ELYRA P.1 equipped with the following laser lines: a HR diode 50 mW 405 nm laser and a HR DPSS 200 mW 561 nm laser and an Andor EM-CCD camera iXon DU 897 camera. Fluorescence was detected using a long pass 570nm filter (LP570), similar to a procedure described before (62).

An alpha Plan-Apochromat 100x/1,46 Oil DIC M27 objective was used for imaging. $100 \mathrm{~nm}$ TetraSpeck microsphere and the implemented drift correction tool were used to check for lateral drift and eventual drift correction. Calculation of the PALM image was performed via the 2D x/y Gaussian fit provided by the Zen2 software (Zeiss) with 
607 the following parameters: a peak mask size of 9 pixels $(1$ pixel $=100 \mathrm{~nm})$ and a peak

608 intensity to noise ratio of 6 (overlapping events were discarded). Z-axis was stabilized using the "Definite Focus" system.

The C. glutamicum strains ptsF::pamCherry-ptsF and ptsG::PAmCherry-linker-ptsG were imaged in the same way for each different condition: fructose, glucose and acetate. During the 10,000 frames that were collected during imaging, PAmCherry was activated using a linear gradient of the $405 \mathrm{~nm}$ laser ranging from $0.01 \%$ to $10 \%$ (the 405 laser power was chosen in a way that minimized conversion of two separate PAmCherry molecules in close proximity at the same time). Converted PAmCherry was imaged using the $561 \mathrm{~nm}$ laser at $15 \%$ (transfer mode) for $50 \mathrm{~ms}$ using an EMCCD gain of 200.

The localization events recognized by the Gaussian fit were filtered for photon number (70-350 photons) and point spread function (PSF) width at 1/e maximum (70$170 \mathrm{~nm}$ ) in order to exclude the localization events originated by background (i.e.: dust particles) and/or the co-occurrence of multiple PAmCherry molecules.

\section{Western Blot and in-gel fluorescence}

For western blot confirmation of the strains, cultures were grown in CGXII with $2 \%$ of the specific Pts sugars as carbon sources to an $\mathrm{OD}_{600}=4$, then harvested by centrifugation and resuspended in $1 \mathrm{~mL}$ of disruption buffer $(100 \mathrm{mM} \mathrm{NaCl}, 100 \mathrm{~mm}$

$627 \mathrm{KCl}$, DNAse, Protease inhibitor). Cells were subsequently disrupted by 10 cycles of $62830 \mathrm{~s}$ at $6 \mathrm{~m} / \mathrm{s}$ in FastPrep 24 (MP Biomedicals). The lysate was then centrifuged 
630

631

632

633

with loading dye and $15 \mu \mathrm{L}$ was loaded on $0.1 \%$ SDS-containing $12 \%$ polyacrylamide gel, separated by electrophoresis and transferred to a PVDF membrane. Western blotting was carried out using standard methods: Blocking step was performed by incubation of the membrane in $10 \mathrm{ml}$ TBS $+5 \%$ milk for $1 \mathrm{~h}$ at RT, the primary antibody anti-mCherry was added in a 1:2000 dilution, followed by incubation for 1 hour. After washing the membrane 3 times with TBS, the secondary anti-rabbit antibody was added diluted $1: 10000$ to $10 \mathrm{ml}$ TBS-T $+5 \%$ milk and incubated membrane for $1 \mathrm{hr}$ at RT. After another washing step, 5-bromo-4chloro-3indolylphosphate and nitroblue tetrazolium solution (NBD/BCIP) was added and the membrane was incubated in the dark until the colors on the membrane were developed.

For in-gel fluorescence of strains containing mNeonGreen fusions, SDS gels cwere analyzed with a Typhoon Trio 9410 (Amersham Biosciences, GE) with 488nm laser excitation of mNeonGreen and fluorescence was detected using a 520nm filter.

\section{Acknowledgements}

We thank Karin Schubert (LMU Munich) for the help with HPLC analysis.

\section{Funding information}

This work was funded with aid of the Brazilian exchange program Science Without Borders (fellowship to G.B.M) and the Ministry of Science and Education (BMBF: 031A302 e:Bio-Modul II: 0.6 plus). We thank the Deutsche 
Forschungsgemeinschaft (INST 86/1583-1) for the ELYRA microscope and (INST 86/1452-1) for the DeltaVision microscope.

\section{Author contributions}

G.B.M., G.G. and M.B. designed the study

G.B.M. performed the genetic manipulations, strain characterization and widefield microscopy analysis

G.G. performed the PALM analysis

G.B.M., G.G. and M.B. analyzed the data and wrote the manuscript.

\section{References}

1. Epps HM, Gale EF. 1942. The influence of the presence of glucose during growth on the enzymic activities of Escherichia coli: comparison of the effect with that produced by fermentation acids. Biochem J 36:619-623.

2. Monod J. 1942. Recherches sur la croissance des cultures bactériennes.

3. Gorke B, Stulke J. 2008. Carbon catabolite repression in bacteria: many ways to make the most out of nutrients. Nat Rev Microbiol 6:613-624.

4. Lengeler JW, Jahreis K. 2009. Bacterial PEP-dependent carbohydrate: phosphotransferase systems couple sensing and global control mechanisms. Contrib Microbiol 16:65-87.

5. Lengeler JW. 2015. PTS 50: Past, Present and Future, or Diauxie Revisited. J Mol Microbiol Biotechnol 25:79-93.

6. Lengeler JW. 2000. Metabolic networks: a signal-oriented approach to cellular models. Biol Chem 381:911-920.

7. Deutscher J, Ake FM, Derkaoui M, Zebre AC, Cao TN, Bouraoui H, Kentache T, Mokhtari A, Milohanic E, Joyet P. 2014. The bacterial phosphoenolpyruvate:carbohydrate phosphotransferase system: regulation by protein phosphorylation and phosphorylationdependent protein-protein interactions. Microbiol Mol Biol Rev 78:231-256.

8. Deutscher J, Francke C, Postma PW. 2006. How phosphotransferase system-related protein phosphorylation regulates carbohydrate metabolism in bacteria. Microbiol Mol Biol Rev 70:939-1031.

9. Frunzke J, Engels V, Hasenbein S, Gatgens C, Bott M. 2008. Co-ordinated regulation of gluconate catabolism and glucose uptake in Corynebacterium glutamicum by two functionally equivalent transcriptional regulators, GntR1 and GntR2. Mol Microbiol 67:305322. 
10. Wendisch VF, de Graaf AA, Sahm H, Eikmanns BJ. 2000. Quantitative determination of metabolic fluxes during coutilization of two carbon sources: comparative analyses with Corynebacterium glutamicum during growth on acetate and/or glucose. J Bacteriol 182:30883096.

11. Shah A, Blombach B, Gauttam R, Eikmanns BJ. 2018. The RamA regulon: complex regulatory interactions in relation to central metabolism in Corynebacterium glutamicum. Appl Microbiol Biotechnol doi:10.1007/s00253-018-9085-3.

12. Wendisch VF. 2017. Microbial Production of Amino Acid-Related Compounds. Adv Biochem Eng Biotechnol 159:255-269.

13. Wendisch VF, Brito LF, Gil Lopez M, Hennig G, Pfeifenschneider J, Sgobba E, Veldmann KH. 2016. The flexible feedstock concept in Industrial Biotechnology: Metabolic engineering of Escherichia coli, Corynebacterium glutamicum, Pseudomonas, Bacillus and yeast strains for access to alternative carbon sources. J Biotechnol 234:139-157.

14. Ikeda M. 2012. Sugar transport systems in Corynebacterium glutamicum: features and applications to strain development. Appl Microbiol Biotechnol 96:1191-1200.

15. Meadow ND, Fox DK, Roseman S. 1990. The bacterial phosphoenolpyruvate: glycose phosphotransferase system. Annu Rev Biochem 59:497-542.

16. Neidhardt FC, Curtiss R. 1996. Escherichia coli and Salmonella : cellular and molecular biology, 2nd ed. ASM Press, Washington, D.C.

17. Cao Y, Jin X, Levin EJ, Huang H, Zong Y, Quick M, Weng J, Pan Y, Love J, Punta M, Rost B, Hendrickson WA, Javitch JA, Rajashankar KR, Zhou M. 2011. Crystal structure of a phosphorylation-coupled saccharide transporter. Nature 473:50-54.

18. Hvorup R, Chang AB, Saier MH, Jr. 2003. Bioinformatic analyses of the bacterial L-ascorbate phosphotransferase system permease family. J Mol Microbiol Biotechnol 6:191-205.

19. Tanaka Y, Okai N, Teramoto H, Inui M, Yukawa H. 2008. Regulation of the expression of phosphoenolpyruvate: carbohydrate phosphotransferase system (PTS) genes in Corynebacterium glutamicum R. Microbiology 154:264-274.

20. Engels V, Wendisch VF. 2007. The DeoR-type regulator SugR represses expression of ptsG in Corynebacterium glutamicum. J Bacteriol 189:2955-2966.

21. Engels V, Lindner SN, Wendisch VF. 2008. The global repressor SugR controls expression of genes of glycolysis and of the L-lactate dehydrogenase LdhA in Corynebacterium glutamicum. J Bacteriol 190:8033-8044.

22. Gaigalat L, Schluter JP, Hartmann M, Mormann S, Tauch A, Puhler A, Kalinowski J. 2007. The DeoR-type transcriptional regulator SugR acts as a repressor for genes encoding the phosphoenolpyruvate:sugar phosphotransferase system (PTS) in Corynebacterium glutamicum. BMC Mol Biol 8:104.

23. Kawamoto H, Morita T, Shimizu A, Inada T, Aiba H. 2005. Implication of membrane localization of target mRNA in the action of a small RNA: mechanism of post-transcriptional regulation of glucose transporter in Escherichia coli. Genes Dev 19:328-338.

24. Maki K, Uno K, Morita T, Aiba H. 2008. RNA, but not protein partners, is directly responsible for translational silencing by a bacterial Hfq-binding small RNA. Proceedings of the National Academy of Sciences 105:10332-10337.

25. Saier MH, Jr., Ramseier TM. 1996. The catabolite repressor/activator (Cra) protein of enteric bacteria. J Bacteriol 178:3411-3417.

26. Chavarría M, Durante-Rodríguez G, Krell T, Santiago C, Brezovsky J, Damborsky J, de Lorenzo V. 2014. Fructose 1-phosphate is the one and only physiological effector of the Cra (FruR) regulator of Pseudomonas putida. FEBS Open Bio 4:377-386.

27. Galinier A, Deutscher J. 2017. Sophisticated Regulation of Transcriptional Factors by the Bacterial Phosphoenolpyruvate: Sugar Phosphotransferase System. J Mol Biol 429:773-789. 
bioRxiv preprint doi: https://doi org/10.1101/349514; this version posted June 18, 2018. The copyright holder for this preprint (which was not certified by peer review) is the author/funder, who has granted bioRxiv a license to display the preprint in perpetuity. It is made available under aCC-BY-NC-ND 4.0 International license.

28. Nothaft H, Parche S, Kamionka A, Titgemeyer F. 2003. In vivo analysis of HPr reveals a fructose-specific phosphotransferase system that confers high-affinity uptake in Streptomyces coelicolor. J Bacteriol 185:929-937.

29. Viana R, Monedero V, Dossonnet V, Vadeboncoeur C, Perez-Martinez G, Deutscher J. 2000. Enzyme I and HPr from Lactobacillus casei: their role in sugar transport, carbon catabolite repression and inducer exclusion. Mol Microbiol 36:570-584.

30. Hasegawa S, Tanaka Y, Suda M, Jojima T, Inui M. 2017. Enhanced Glucose Consumption and Organic Acid Production by Engineered Corynebacterium glutamicum Based on Analysis of a pfkB1 Deletion Mutant. Appl Environ Microbiol 83.

31. Maddock JR, Shapiro L. 1993. Polar location of the chemoreceptor complex in the Escherichia coli cell. Science 259:1717-1723.

32. Lopian L, Elisha Y, Nussbaum-Shochat A, Amster-Choder O. 2010. Spatial and temporal organization of the E. coli PTS components. EMBO J 29:3630-3645.

33. Govindarajan S, Elisha Y, Nevo-Dinur K, Amster-Choder O. 2013. The general phosphotransferase system proteins localize to sites of strong negative curvature in bacterial cells. MBio 4:e00443-00413.

34. Kawaguchi H, Yoshihara K, Hara KY, Hasunuma T, Ogino C, Kondo A. 2018. Metabolome analysis-based design and engineering of a metabolic pathway in Corynebacterium glutamicum to match rates of simultaneous utilization of D-glucose and L-arabinose. Microb Cell Fact 17:76.

35. Prosser GA, de Carvalho LP. 2013. Kinetic mechanism and inhibition of Mycobacterium tuberculosis D-alanine:D-alanine ligase by the antibiotic D-cycloserine. FEBS J 280:1150-1166.

36. Parche S, Burkovski A, Sprenger GA, Weil B, Kramer R, Titgemeyer F. 2001. Corynebacterium glutamicum: a dissection of the PTS. J Mol Microbiol Biotechnol 3:423-428.

37. Mercier R, Kawai Y, Errington J. 2014. General principles for the formation and proliferation of a wall-free (L-form) state in bacteria. Elife $\mathbf{3}$.

38. Greenfield D, McEvoy AL, Shroff H, Crooks GE, Wingreen NS, Betzig E, Liphardt J. 2009. Selforganization of the Escherichia coli chemotaxis network imaged with super-resolution light microscopy. PLoS Biol 7:e1000137.

39. Rohwer JM, Postma PW, Kholodenko BN, Westerhoff HV. 1998. Implications of macromolecular crowding for signal transduction and metabolite channeling. Proc Natl Acad Sci U S A 95:10547-10552.

40. Norris V, Gascuel P, Guespin-Michel J, Ripoll C, Saier MH, Jr. 1999. Metabolite-induced metabolons: the activation of transporter-enzyme complexes by substrate binding. Mol Microbiol 31:1592-1595.

41. Nijland JG, Vos E, Shin HY, de Waal PP, Klaassen P, Driessen AJ. 2016. Improving pentose fermentation by preventing ubiquitination of hexose transporters in Saccharomyces cerevisiae. Biotechnol Biofuels 9:158.

42. Zhuang K, Vemuri GN, Mahadevan R. 2011. Economics of membrane occupancy and respirofermentation. Mol Syst Biol 7:500.

43. Donovan C, Bramkamp M. 2009. Characterization and subcellular localization of a bacterial flotillin homologue. Microbiology 155:1786-1799.

44. Lopez D, Kolter R. 2010. Functional microdomains in bacterial membranes. Genes Dev 24:1893-1902.

45. Erhardt H, Dempwolff F, Pfreundschuh M, Riehle M, Schafer C, Pohl T, Graumann P, Friedrich T. 2014. Organization of the Escherichia coli aerobic enzyme complexes of oxidative phosphorylation in dynamic domains within the cytoplasmic membrane. Microbiologyopen 3:316-326.

46. Llorente-Garcia I, Lenn T, Erhardt H, Harriman OL, Liu LN, Robson A, Chiu SW, Matthews S, Willis NJ, Bray CD, Lee SH, Shin JY, Bustamante C, Liphardt J, Friedrich T, Mullineaux CW, 
Leake MC. 2014. Single-molecule in vivo imaging of bacterial respiratory complexes indicates delocalized oxidative phosphorylation. Biochim Biophys Acta 1837:811-824.

47. Bach JN, Bramkamp M. 2013. Flotillins functionally organize the bacterial membrane. Mol Microbiol 88:1205-1217.

48. Lux R, Jahreis K, Bettenbrock K, Parkinson JS, Lengeler JW. 1995. Coupling the phosphotransferase system and the methyl-accepting chemotaxis protein-dependent chemotaxis signaling pathways of Escherichia coli. Proc Natl Acad Sci U S A 92:11583-11587.

49. Lux R, Munasinghe VR, Castellano F, Lengeler JW, Corrie JE, Khan S. 1999. Elucidation of a PTS-carbohydrate chemotactic signal pathway in Escherichia coli using a time-resolved behavioral assay. Mol Biol Cell 10:1133-1146.

50. Besschetnova TY, Montefusco DJ, Asinas AE, Shrout AL, Antommattei FM, Weis RM. 2008. Receptor density balances signal stimulation and attenuation in membrane-assembled complexes of bacterial chemotaxis signaling proteins. Proc Natl Acad Sci U S A 105:1228912294.

51. Lamanna AC, Ordal GW, Kiessling LL. 2005. Large increases in attractant concentration disrupt the polar localization of bacterial chemoreceptors. Mol Microbiol 57:774-785.

52. Veldhuis G, Hink M, Krasnikov V, van den Bogaart G, Hoeboer J, Visser AJ, Broos J, Poolman B. 2006. The oligomeric state and stability of the mannitol transporter, Enzymell( $\mathrm{mtl})$, from Escherichia coli: a fluorescence correlation spectroscopy study. Protein Sci 15:1977-1986.

53. Chen Q, Amster-Choder O. 1998. BgIF, the sensor of the bgl system and the beta-glucosides permease of Escherichia coli: evidence for dimerization and intersubunit phosphotransfer. Biochemistry 37:8714-8723.

54. Rohwer JM, Meadow ND, Roseman S, Westerhoff HV, Postma PW. 2000. Understanding glucose transport by the bacterial phosphoenolpyruvate:glycose phosphotransferase system on the basis of kinetic measurements in vitro. J Biol Chem 275:34909-34921.

55. Francke C, Postma PW, Westerhoff HV, Blom JG, Peletier MA. 2003. Why the phosphotransferase system of Escherichia coli escapes diffusion limitation. Biophys J 85:612622.

56. Francke C, Westerhoff HV, Blom JG, Peletier MA. 2002. Flux control of the bacterial phosphoenolpyruvate:glucose phosphotransferase system and the effect of diffusion. Mol Biol Rep 29:21-26.

57. Evans GA. Molecular cloning: A laboratory manual. Second edition. Volumes 1, 2, and 3. Current protocols in molecular biology. Volumes 1 and 2. Cell 61:17-18.

58. Keilhauer C, Eggeling L, Sahm H. 1993. Isoleucine synthesis in Corynebacterium glutamicum: molecular analysis of the ilvB-ilvN-ilvC operon. J Bacteriol 175:5595-5603.

59. Sieger B, Schubert K, Donovan C, Bramkamp M. 2013. The lipid II flippase RodA determines morphology and growth in Corynebacterium glutamicum. Mol Microbiol 90:966-982.

60. Schindelin J, Arganda-Carreras I, Frise E, Kaynig V, Longair M, Pietzsch T, Preibisch S, Rueden C, Saalfeld S, Schmid B, Tinevez JY, White DJ, Hartenstein V, Eliceiri K, Tomancak P, Cardona A. 2012. Fiji: an open-source platform for biological-image analysis. Nat Methods 9:676-682.

61. Gavet O, Pines J. 2010. Progressive activation of CyclinB1-Cdk1 coordinates entry to mitosis. Dev Cell 18:533-543.

62. Bach JN, Giacomelli G, Bramkamp M. 2017. Sample Preparation and Choice of Fluorophores for Single and Dual Color Photo-Activated Localization Microscopy (PALM) with Bacterial Cells. Methods Mol Biol 1563:129-141. 


\begin{tabular}{lcccc}
\hline & Fructose & Glucose & Acetate & L-Forms \\
\hline PtsF & $2.4(\mathrm{~b})$ & $2.6(\mathrm{a})$ & $2.9(\mathrm{a})$ & $3.3(\mathrm{c})$ \\
PtsG & $2.8(\mathrm{a})$ & $2.5(\mathrm{~b})$ & $2.8(\mathrm{a})$ & $3.3(\mathrm{c})$ \\
\hline
\end{tabular}

Table 1. Growth rates and glucose consumption rates. Growth rate $\left[h^{-1}\right]$ and substrate uptake [g g-1 h-1] of RES 167 WT and strains CGM001, CGM002, CGM003 and CGM005 in CGXII supplemented with glucose and fructose.

\begin{tabular}{lcccc}
\hline & \multicolumn{2}{c}{ Glucose } & \multicolumn{2}{c}{ Fructose } \\
\hline & $\begin{array}{c}\text { growth rate } \\
{\left[\mathrm{h}^{-1}\right]}\end{array}$ & $\begin{array}{c}\text { uptake (qs) } \\
{\left[\mathrm{g} \mathrm{g}^{-1} \mathrm{~h}^{-1}\right]}\end{array}$ & $\begin{array}{c}\text { growth rate } \\
{\left[\mathrm{h}^{-1}\right]}\end{array}$ & $\begin{array}{c}\text { uptake (qs) } \\
{\left[\mathrm{g} \mathrm{g}^{-1} \mathrm{~h}^{-1}\right]}\end{array}$ \\
\hline WT & 0.285 & $0.23 \pm 0.003$ & 0.289 & $0.14 \pm 0.005$ \\
CGM001 & - & - & 0.286 & $0.17 \pm 0.004$ \\
CGM002 & 0.280 & $0.23 \pm 0.001$ & - & - \\
CGM003 & 0.290 & $0.23 \pm 0.001$ & 0.280 & $0.17 \pm 0.004$ \\
CGM005 & 0.285 & $0.23 \pm 0.001$ & - & - \\
\hline
\end{tabular}

Table 2. Number of PTS Ell foci per cell in various growth conditions. Mean values of foci number per cell of mCherry-PtsF and mNeonGreen-PtsG. "Fructose", "Glucose" and "Acetate" represent rod-shaped cells in CGXII medium with the respective carbon sources. "L-Forms" represent L-Form cells in MXM/CGXII supplemented with the transported sugar. Significant statistical differences according to multiple comparison tests after Kruskal-Wallis are represented as letters next to each value.

Table 3. PtsF and PtsG cover different membrane surface areas. Mean values of foci area $\left[\mu \mathrm{m}^{2}\right]$ of mCherry-PtsF and mNeonGreen-PtsG. "Fructose", "Glucose" and

847 "Acetate" represent rod-shaped cells in CGXII medium with different carbon sources. 
sugar. Values are in $\mu \mathrm{m}^{2}$. Significant statistical differences according to Multiple comparison tests after Kruskal-Wallis are represented as letters next to each value.

\begin{tabular}{lcccc}
\hline & Fructose & Glucose & Acetate & L-Forms \\
\hline PtsF & $0.35(\mathrm{~b})$ & $0.18(\mathrm{a})$ & $0.24(\mathrm{a})$ & $0.71(\mathrm{c})$ \\
PtsG & $0.22(\mathrm{a})$ & $0.70(\mathrm{~b})$ & $0.17(\mathrm{c})$ & $1.00(\mathrm{~d})$ \\
\hline
\end{tabular}

852 Table 4. PTS Ell complex surface coverage. Foci area/cell area ratio of mCherry853 PtsF and mNeonGreen-PtsG. "Fructose", "Glucose" and "Acetate" represent rod854 shaped cells in CGXII medium with different carbon sources. "L-Forms" represent L855 Form cells in MXM/CGXII supplemented with the transported sugar. Significant 856 statistical differences according to multiple comparison tests after Kruskal-Wallis are 857 represented as letters next to each value.

\begin{tabular}{lcccc}
\hline & Fructose & Glucose & Acetate & L-Forms \\
\hline PtsF & $0.10(\mathrm{~b})$ & $0.05(\mathrm{a})$ & $0.06(\mathrm{a})$ & $0.08(\mathrm{c})$ \\
PtsG & $0.06(\mathrm{c})$ & $0.20(\mathrm{~b})$ & $0.04(\mathrm{a})$ & $0.11(\mathrm{c})$ \\
\hline
\end{tabular}




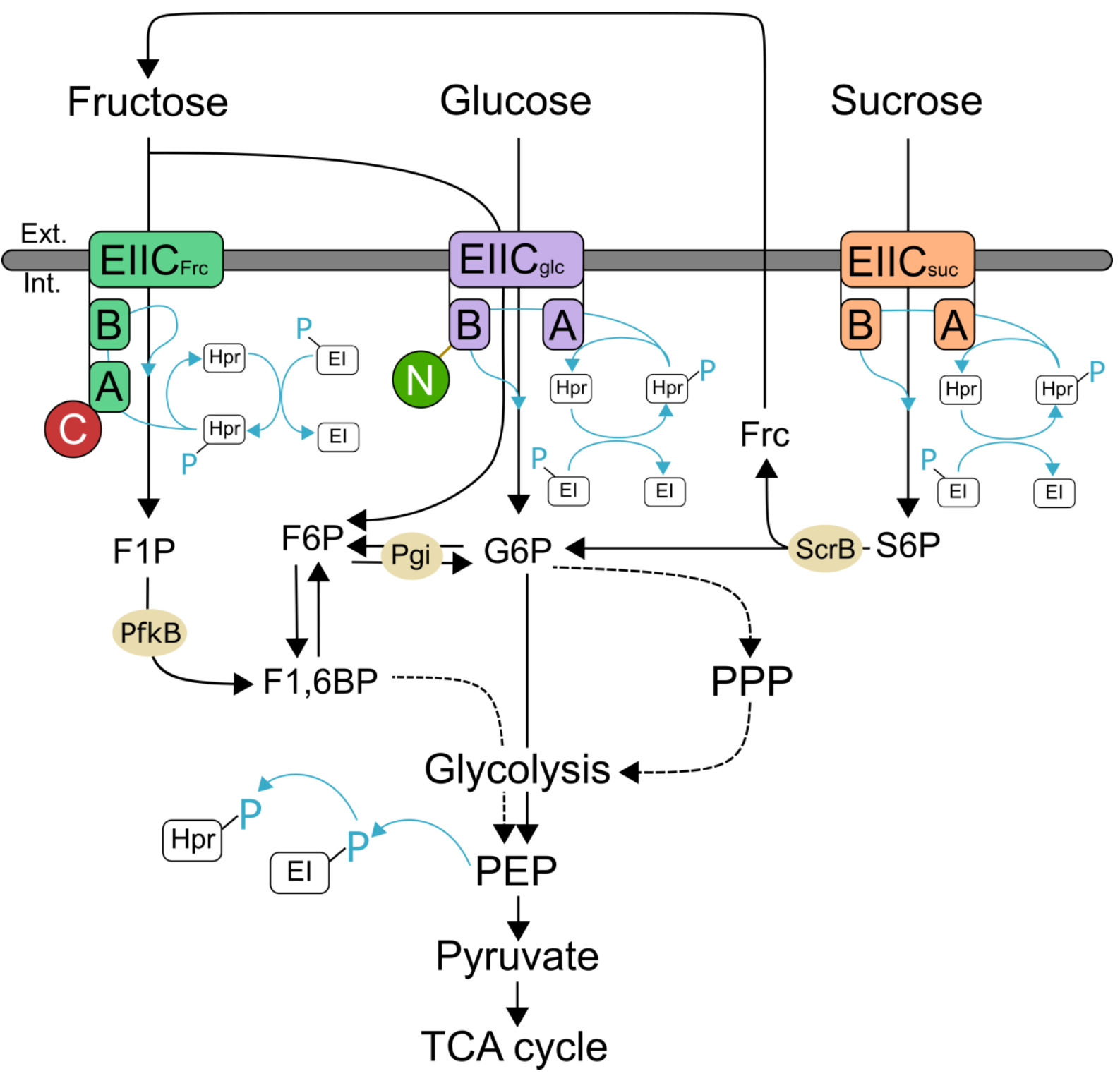

Figure 1. Schematic view of the sugar uptake mediated by the phosphoenolpyruvate-dependent phosphotransferase systems (PTS) in

866 Corynebacterium glutamicum. Blue lines indicate phosphoryl group $\left(\mathrm{PO}_{3}^{2-}\right)$

867 transfer. Fluorescent proteins added via allelic replacement represented as C for 
869 PtsG. F1P fructose-1-phosphate, F6P fructose-6-phosphate, F1,6BP fructose-1,6-

870 biphosphate, G6P glucose-6-phosphate, Frc fructose, S6P sucrose-6-phosphate,

871 PPP Pentose phosphate pathway, PEP phosphoenolpyruvate, scrB beta-

872 fructofuranosidase (putative S6P hydrolase), pgi glucose-6-phosphate isomerase,

873 PfkB 1-phosphofructokinase.

874

875 
A

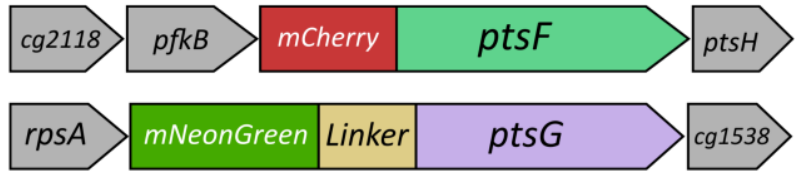
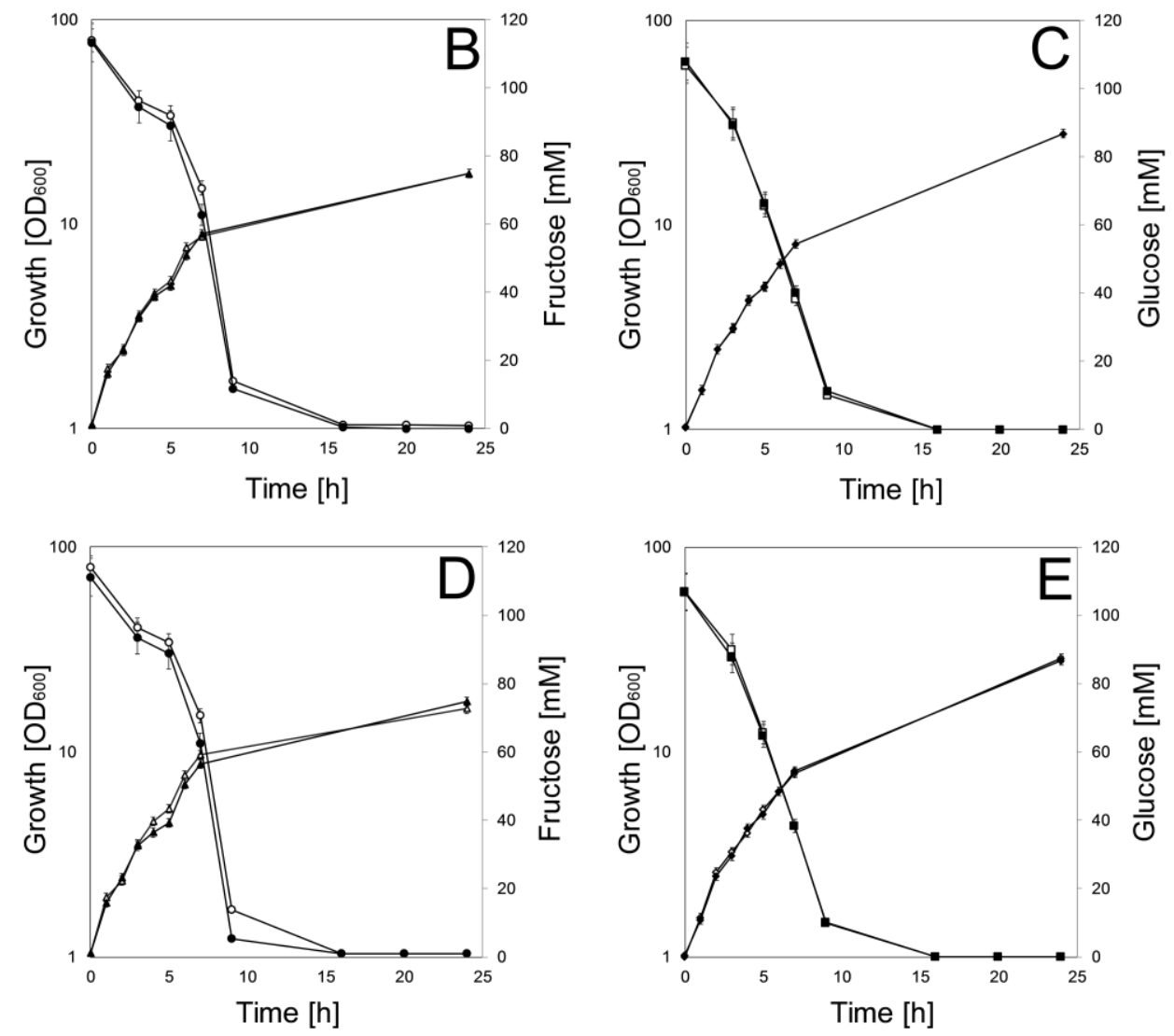

877

Figure 2. Fluorescent fusions of PtsF and PtsG are fully functional. (A) Genetic arrangement of fluorescent fusions constructed via allelic replacement of ptsF::mCherry-ptsF and ptsG::mNeonGreen-linker-ptsG. Growth and sugar

881 consumption of C. glutamicum strains CGM001, CGM002 and CGM003 (filled symbols) versus wild type RES 167 (open symbols) on CGXII containing (B, D) 100 $\mathrm{mM}$ fructose and (C, E) $100 \mathrm{mM}$ glucose. Fructose consumption (circles), glucose consumption (squares), growth on fructose (triangles), and growth on glucose 
bioRxiv preprint doi: https://doi.org/10.1101/349514; this version posted June 18,2018 . The copyright holder for this preprint (which was not certified by peer review) is the author/funder, who has granted bioRxiv a license to display the preprint in perpetuity. It is made available under aCC-BY-NC-ND 4.0 International license.

885 (diamonds) are indicated. Each point represents biological triplicates and standard

886 deviation is indicated.

887 


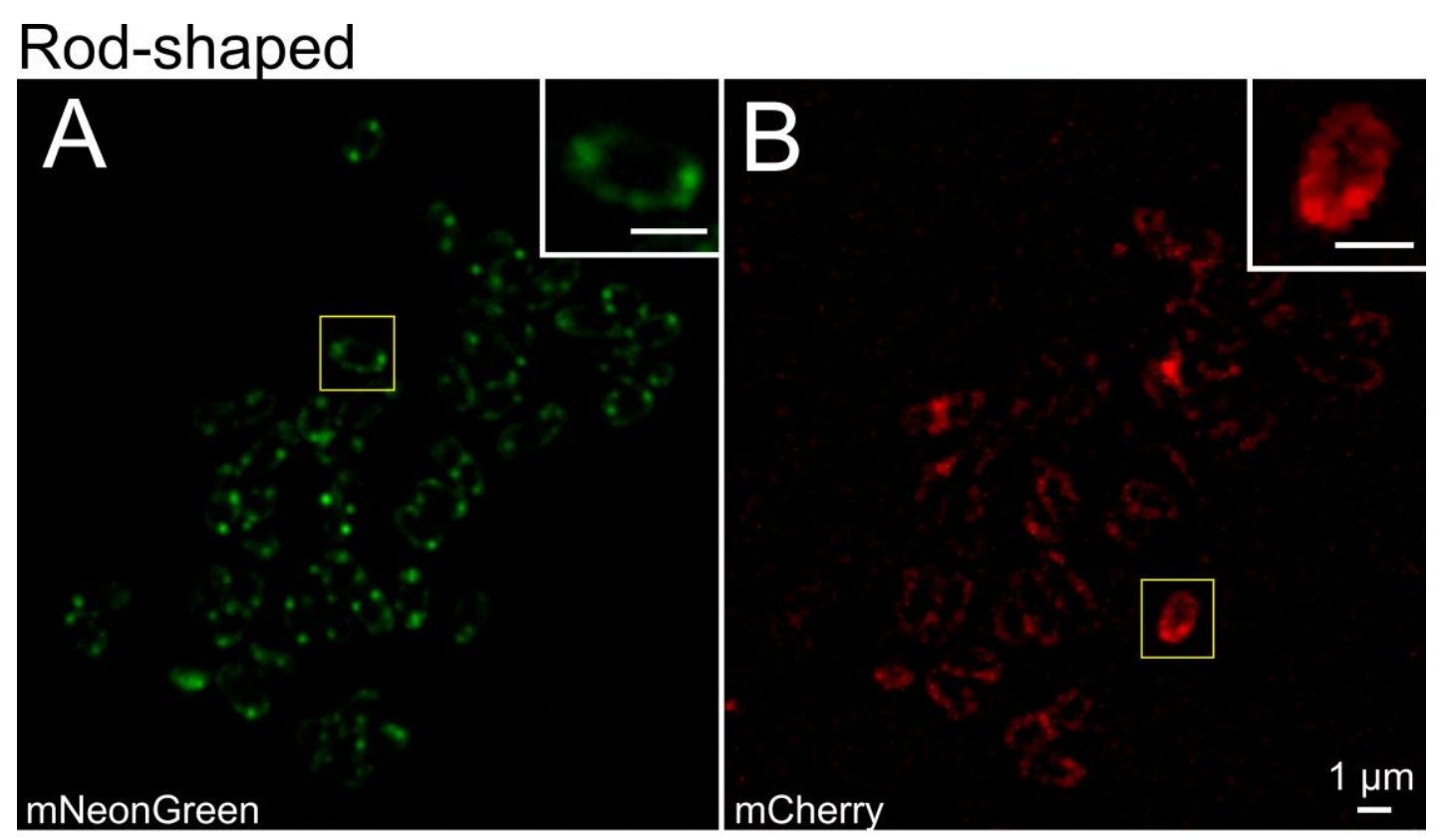

\section{L-Forms}
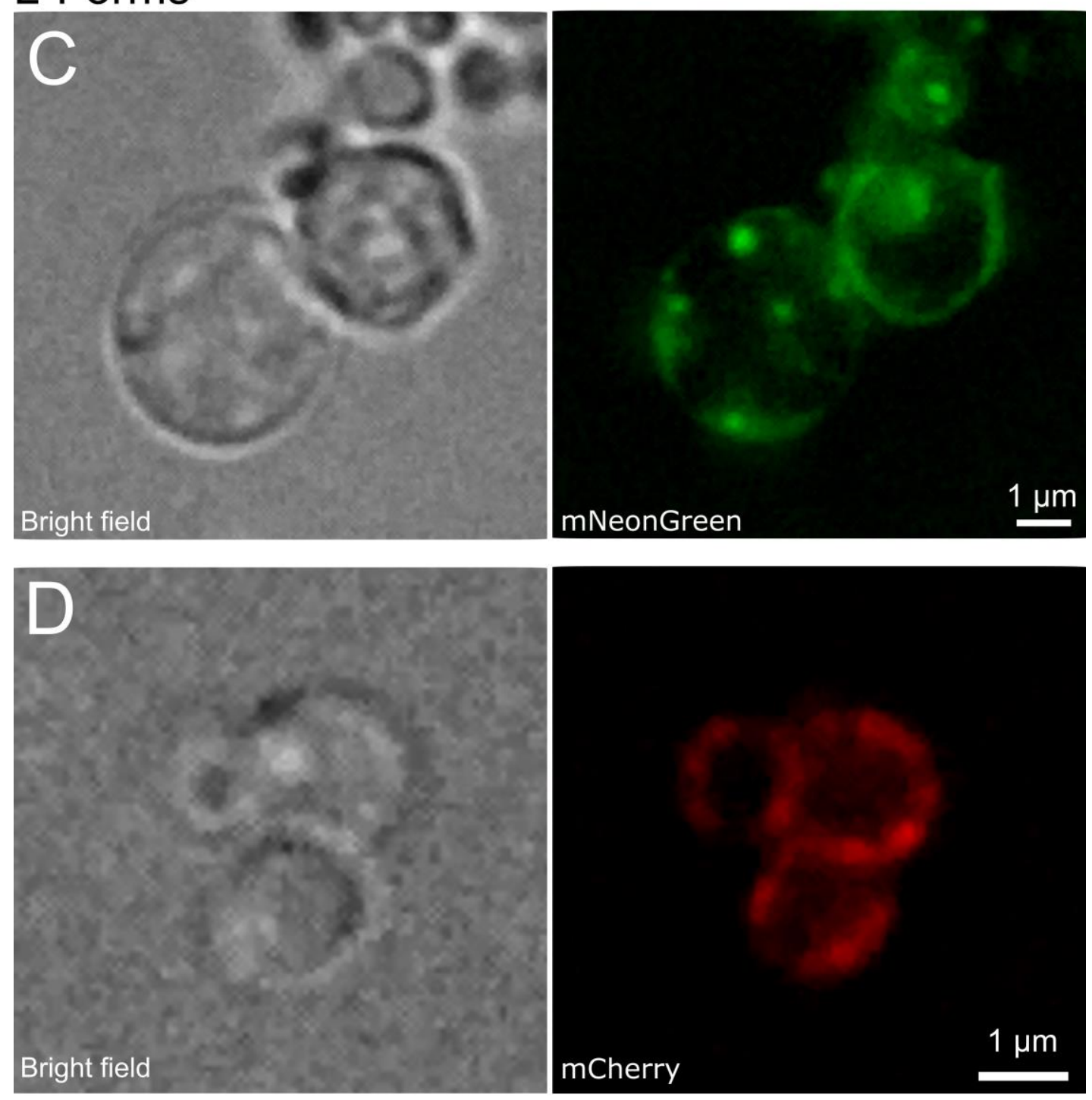
890 Figure 3. PtsF and PtsG localize as clusters distributed along cytoplasmic

891 membrane. Fluorescence microscopy images of C. glutamicum expressing mCherry-

892 PtsF and mNeonGreen-PtsG under different growth conditions: Rod-shaped cells

893 grown in CGXII with (A) glucose and (B) fructose as sole carbon sources. L-Form

894 cells grown in MXM/CGXII with (C) glucose and (D) fructose as sole carbon sources

895 in presence of $200 \mu \mathrm{g} / \mathrm{ml}$ of DCS. 


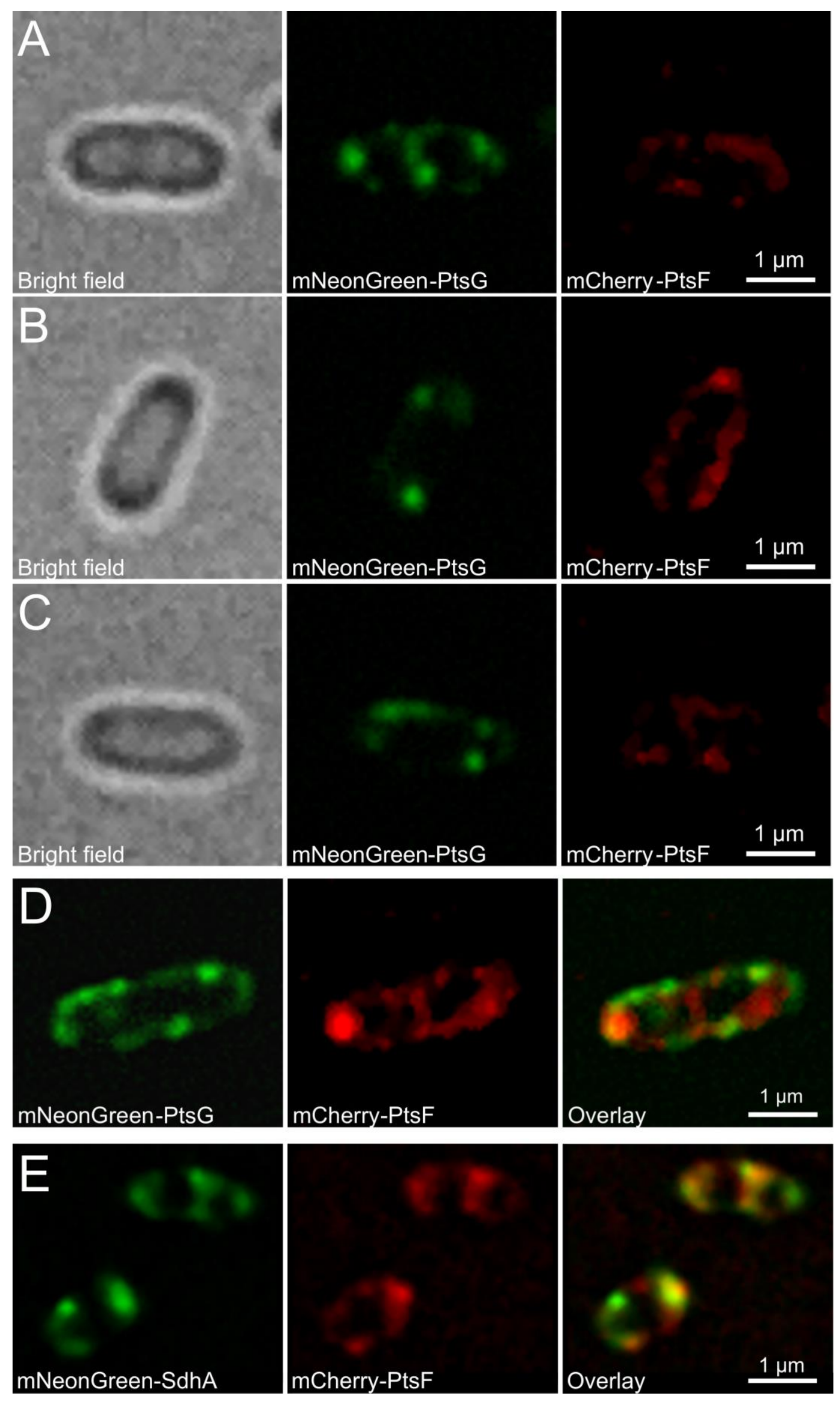


899 Figure 4. PtsF and PtsG clustering changes upon presence or absence of

900 transported substrate. Fluorescence microscopy images of C. glutamicum

901 expressing mCherry-PtsF and mNeonGreen-PtsG under different growth conditions.

902 Cells grown in CGXII with 2\% (A) glucose, (B) fructose or (C) acetate as sole carbon

903 sources. (D) mNeonGreen-PtsG and mCherry-PtsF in CGXII with $2 \%$ glucose and

904 fructose. (E) mNeonGreen-SdhA and mCherry-PtsF in CGXII with and fructose. 

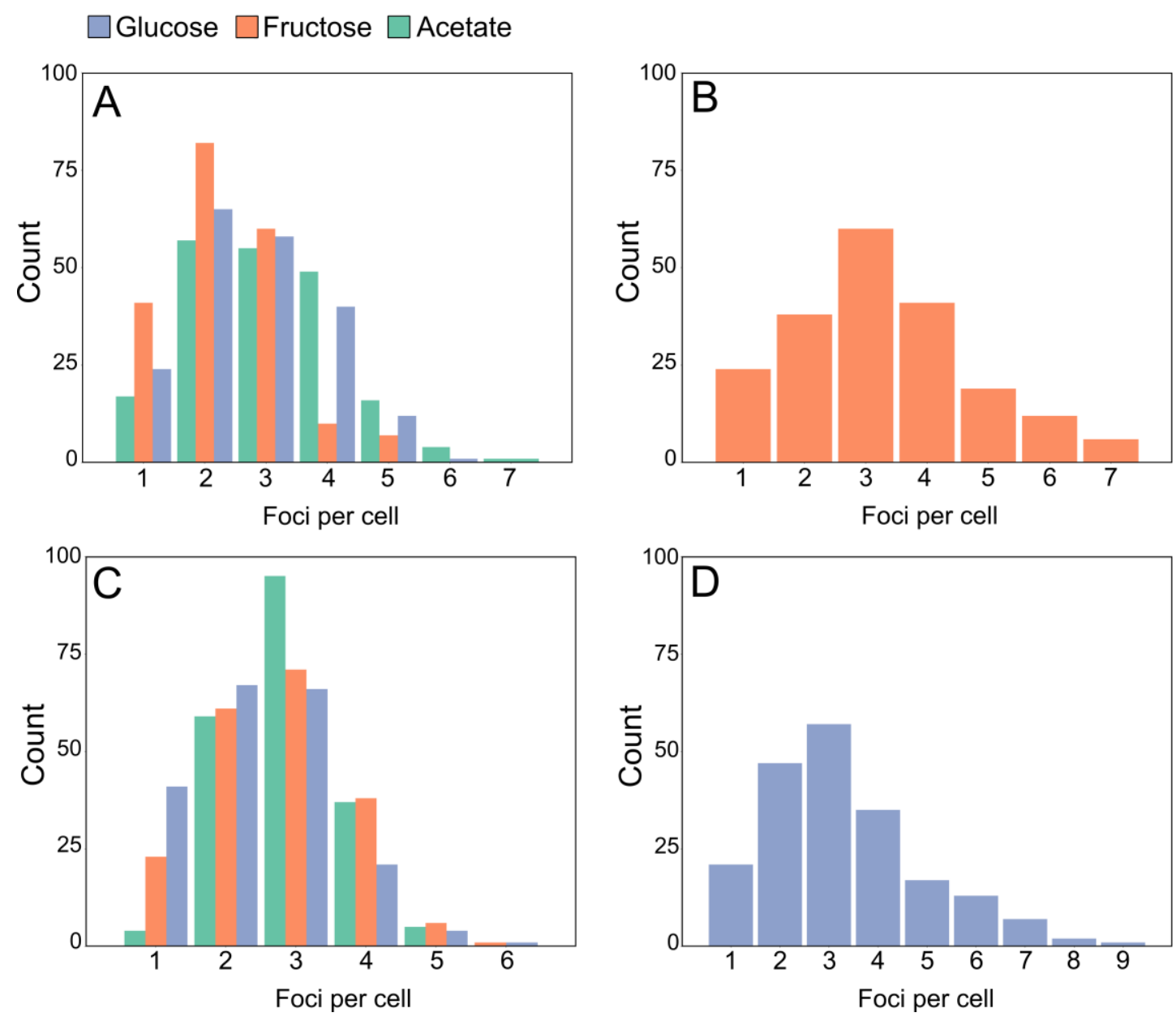

Figure 5. mCherry-PtsF and mNeonGreen-PtsG foci number decreases in presence of transported sugar. Number of foci per cell of $(A)$ mCherry-PtsF in rod shaped cells, (B) mCherry-PtsF in L-form cells. (C) mNeonGreen-PtsG in rod shaped cells, (D) mNeonGreen-PtsG in L-form cells. 
A PtsG-mNeonGreen

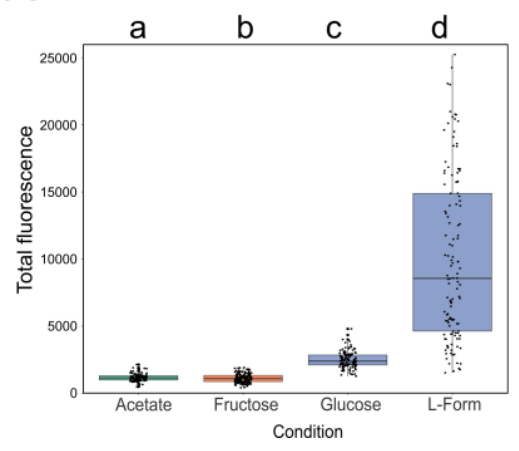

B PtsF-mCherry

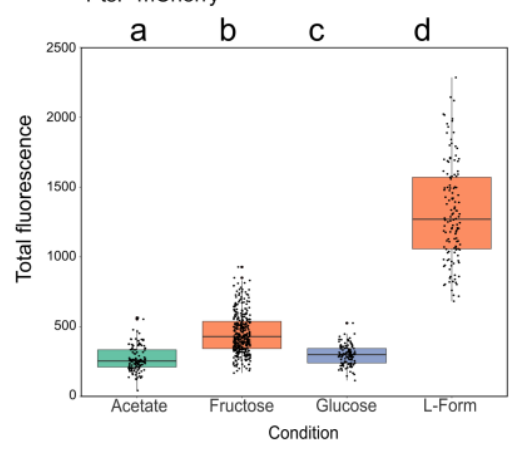

sugar. Corrected total fluorescence of $(A)$ mNeonGreen-PtsG and (B) mCherry-PtsF.

"Fructose", "Glucose" and "Acetate" represent rod-shaped cells in CGXII medium with the respective carbon sources. "L-form" represents L-form cells in MXM/CGXII supplemented with fructose for PtsF and glucose for PtsG. Significant statistical represented as letters above each graph. 
$\square$ Glucose $\square$ Fructose $\square$ Acetate

A

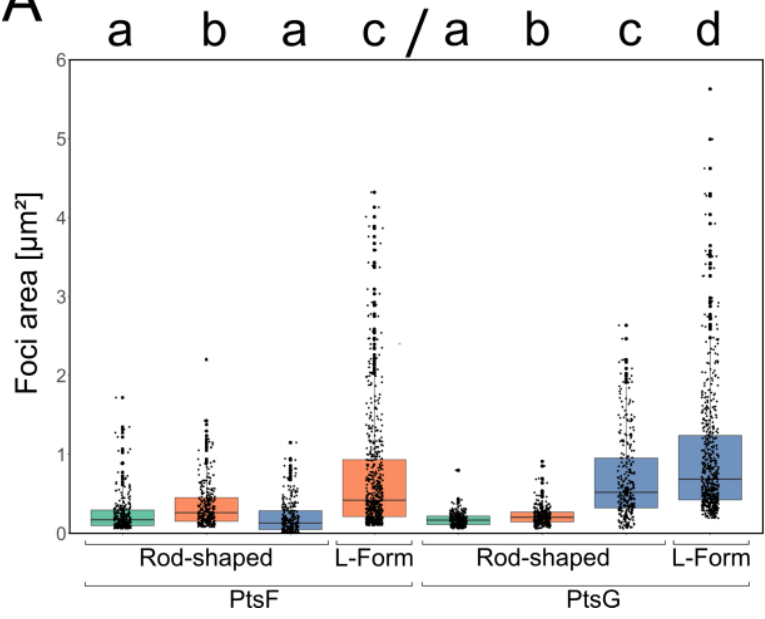

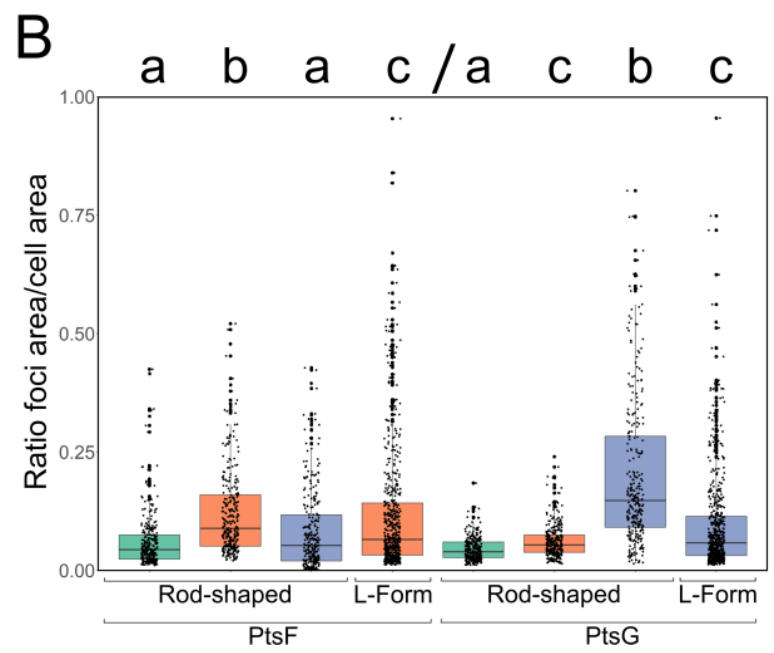

Figure 7. mCherry-PtsF and mNeonGreen-PtsG foci area increases upon presence of transported sugar. (A) mCherry-PtsF and mNeonGreen-PtsG fluorescent foci area in rod-shaped and L-Form cells in CGXII supplemented with different carbon sources (B) Ratio foci area and cell area under different carbon sources and cell shapes. "L-Form" represent L-Form cells in MXM/CGXII supplemented with the transported sugar. Significant statistical differences according to multiple comparison tests after Kruskal-Wallis are represented as letters above each graph. Different letters indicate differences within the same PTS protein. 
A
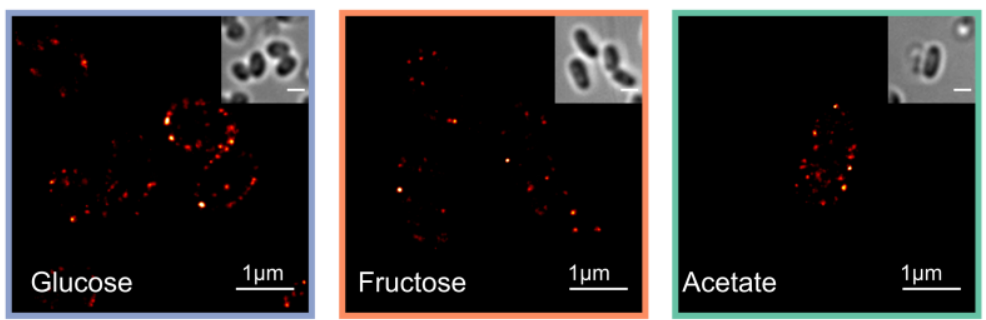

B
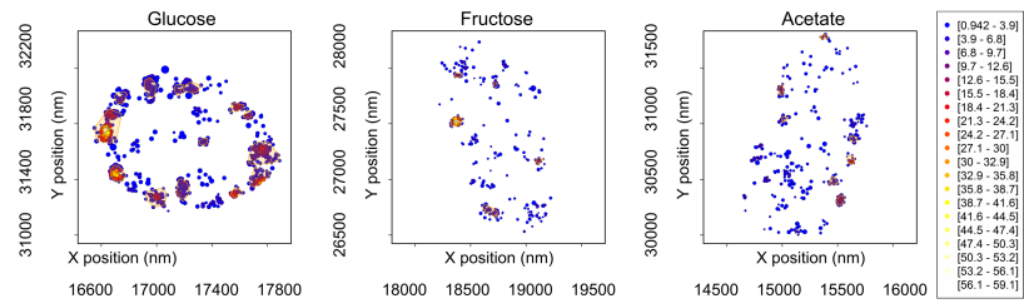

C
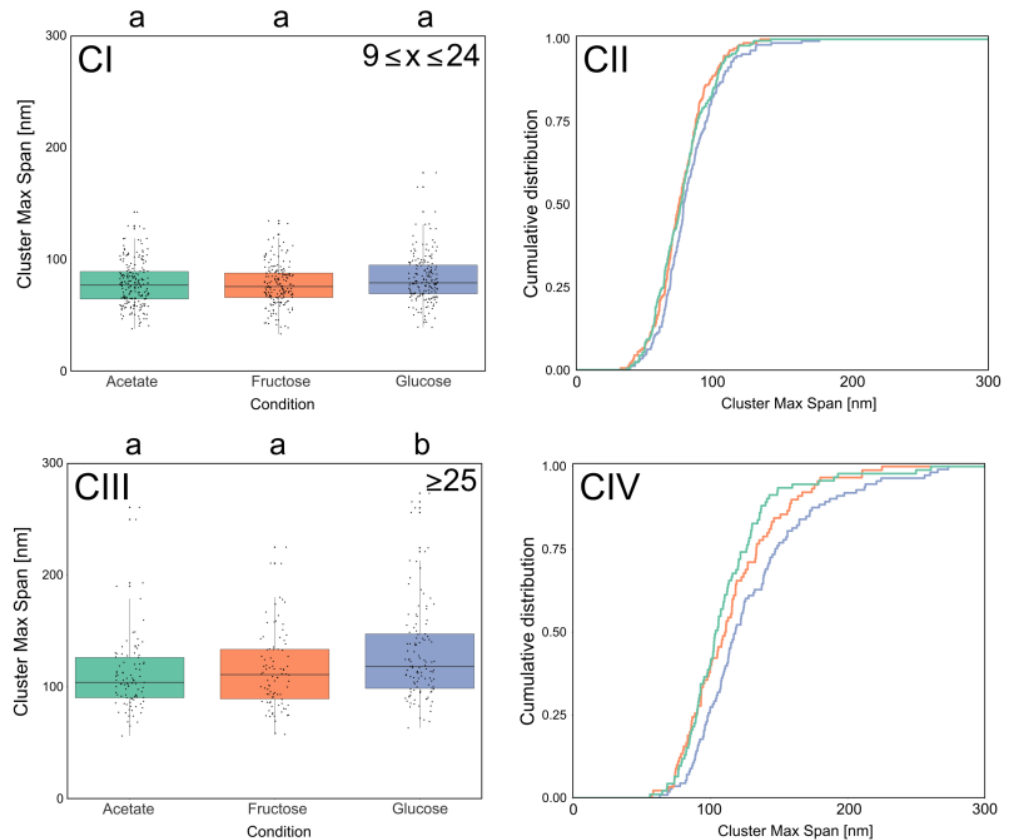

D
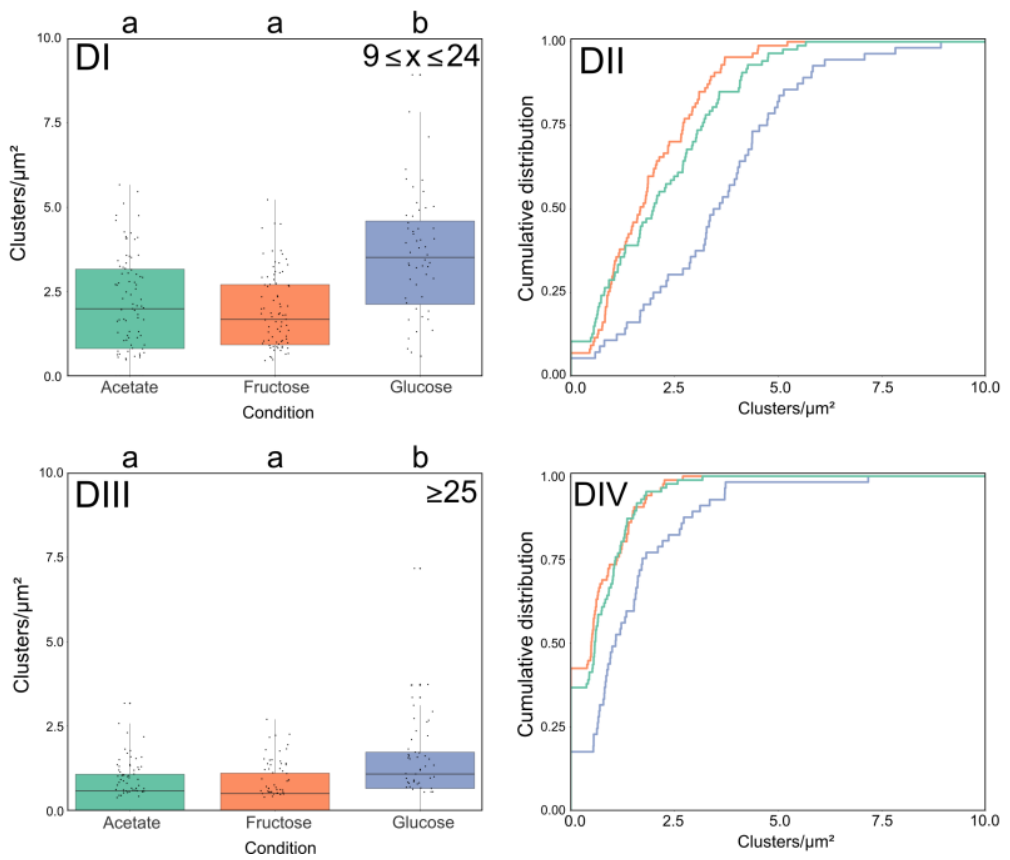
Figure 8. Single molecule localization microscopy reveals dynamic

PAmCherry-PtsG clustering. (A) Super resolution images of $C$. glutamicum strain

938 CGM005 expressing PAmCherry-PtsG under different growth conditions. Cells were

939 grown in CGXII with indicated carbon source. Insets show transmitted light images to

940 see cell outlines. Scale bar is $1 \mu \mathrm{m}$. (B) Plot of detected single molecule localization

941 events localization and clusters identification for a representative cell for each tested

942 condition. Localizations are color coded according to the local event density while the

943 plotted radius is indicating localization precision. The identified clusters are

944 highlighted by orange contours. (C) PtsG clusters maximum span increase in

945 presence of glucose, (D) PtsG clusters are more abundant in presence of glucose.

946 Each parameter is analyzed via (Cl, CIII, DI, DIII) boxplot and (CII, CIV, DII, DIV)

947 cumulative distribution function. Clusters composed of (Cl, CII, DI, DII) $9 \leq x \leq 24$

948 events, and (CIII, CIV, DIII, DIV) $>25$ events were analyzed. Significant statistical

949 differences according to multiple comparison tests after Kruskal-Wallis are

950 represented as letters above each graph. Different letters indicate statistical

951 differences within the same PTS protein. 
A
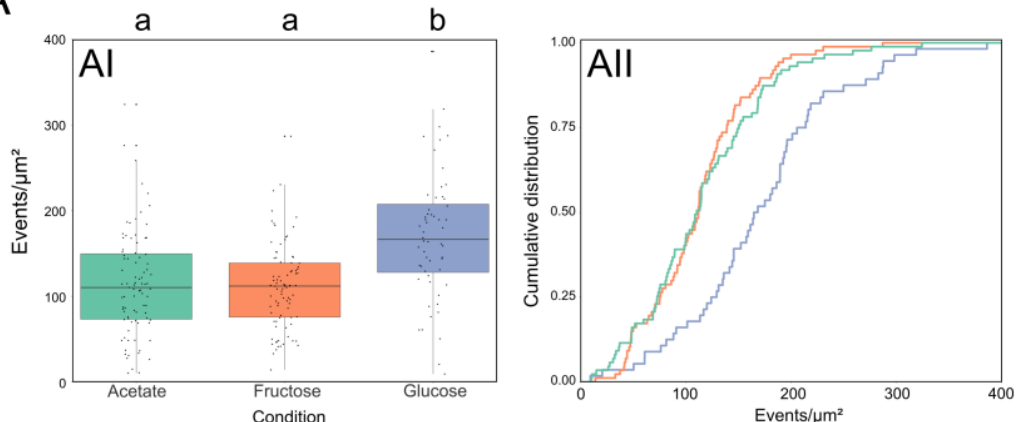

B
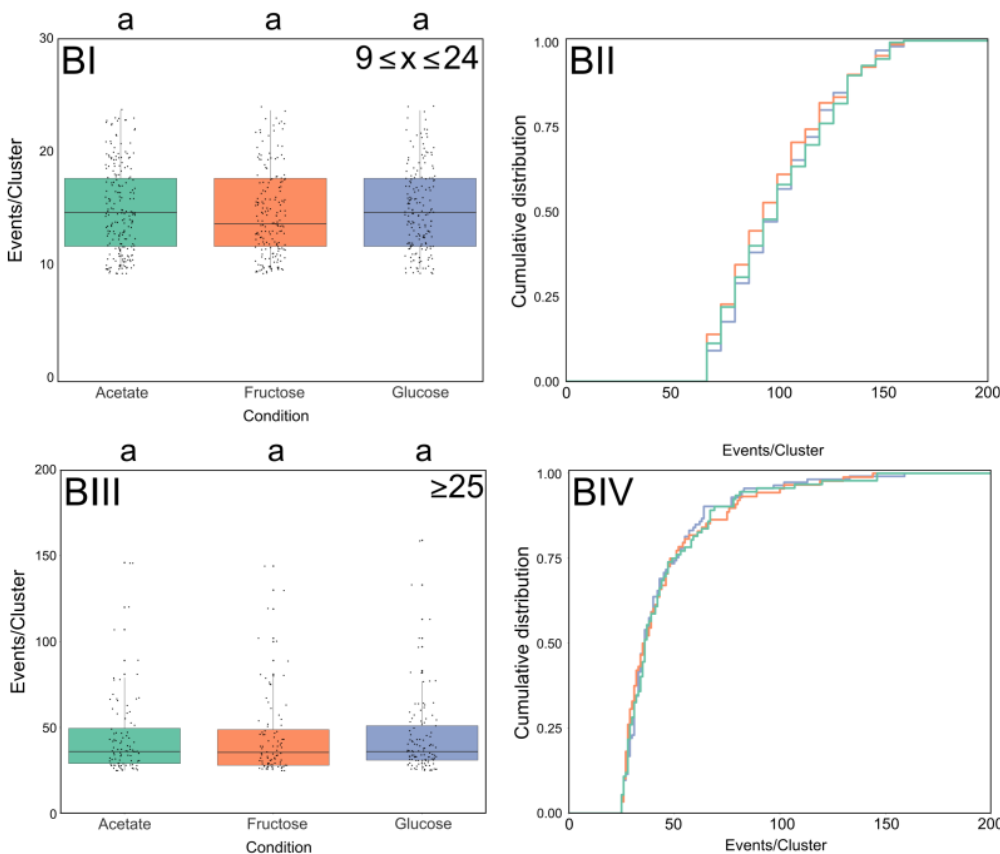

C
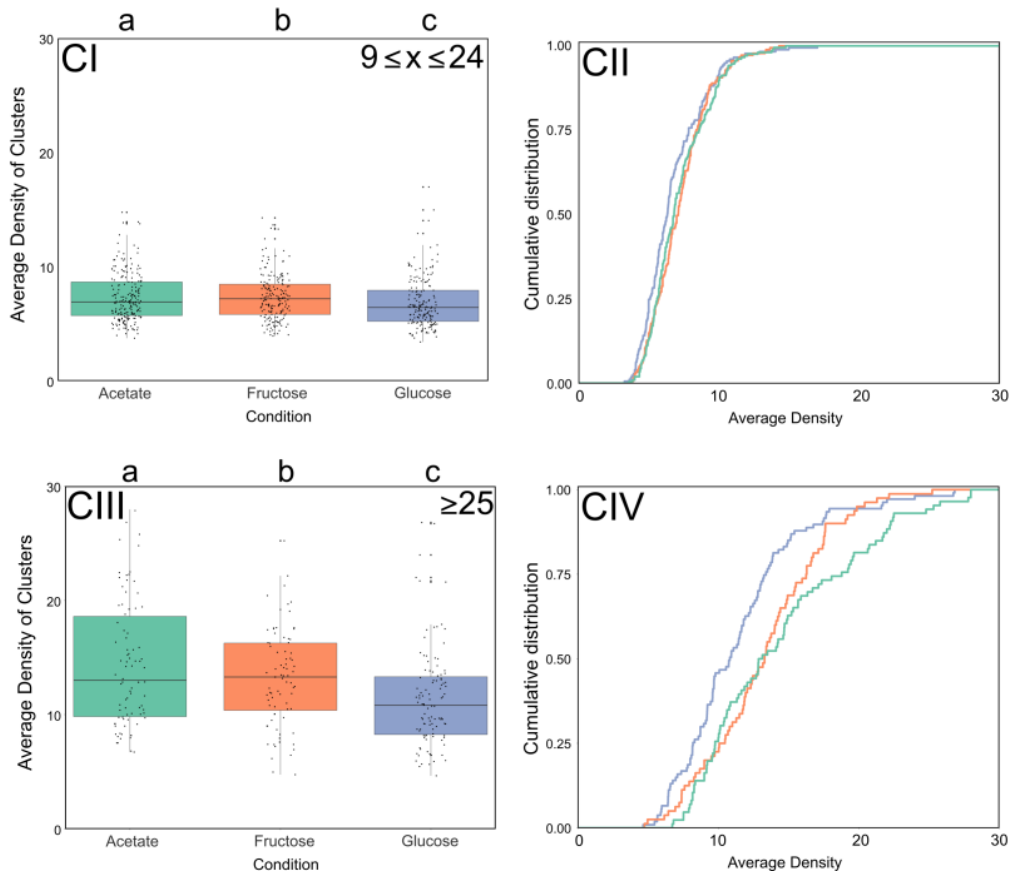


\section{Figure 9. PTS cluster decrease protein density in presence of the transport}

956 substrate. Super resolution PALM data of CGM005 strain grown in CGXII 957 supplemented with $2 \%$ of the indicated carbon sources (A) PtsG number of events 958 per $\mu m^{2}$ increases in presence of glucose, (B) PtsG clusters are composed by the 959 same amount of molecules regardless of the carbon source. Plots represent events 960 per cluster in clusters composed of $>25$ molecules. (C) PtsG clusters have lower 961 average density in presence of glucose. Each parameter is analyzed via (AI, BI, BIII, $962 \mathrm{CI}, \mathrm{CIII})$ boxplot and (AII, BII, BIV, CII, CIV) cumulative distribution function. Clusters composed of (BI, BII, CI, CII) $9 \leq x \leq 24$ events, and (BIII, BIV, CIII, CIV) $>25$ events

964 were analyzed. Significant statistical differences according to multiple comparison 965 tests after Kruskal-Wallis are represented as letters above each graph. Different 966 letters indicate statistical differences within the same PTS protein. 

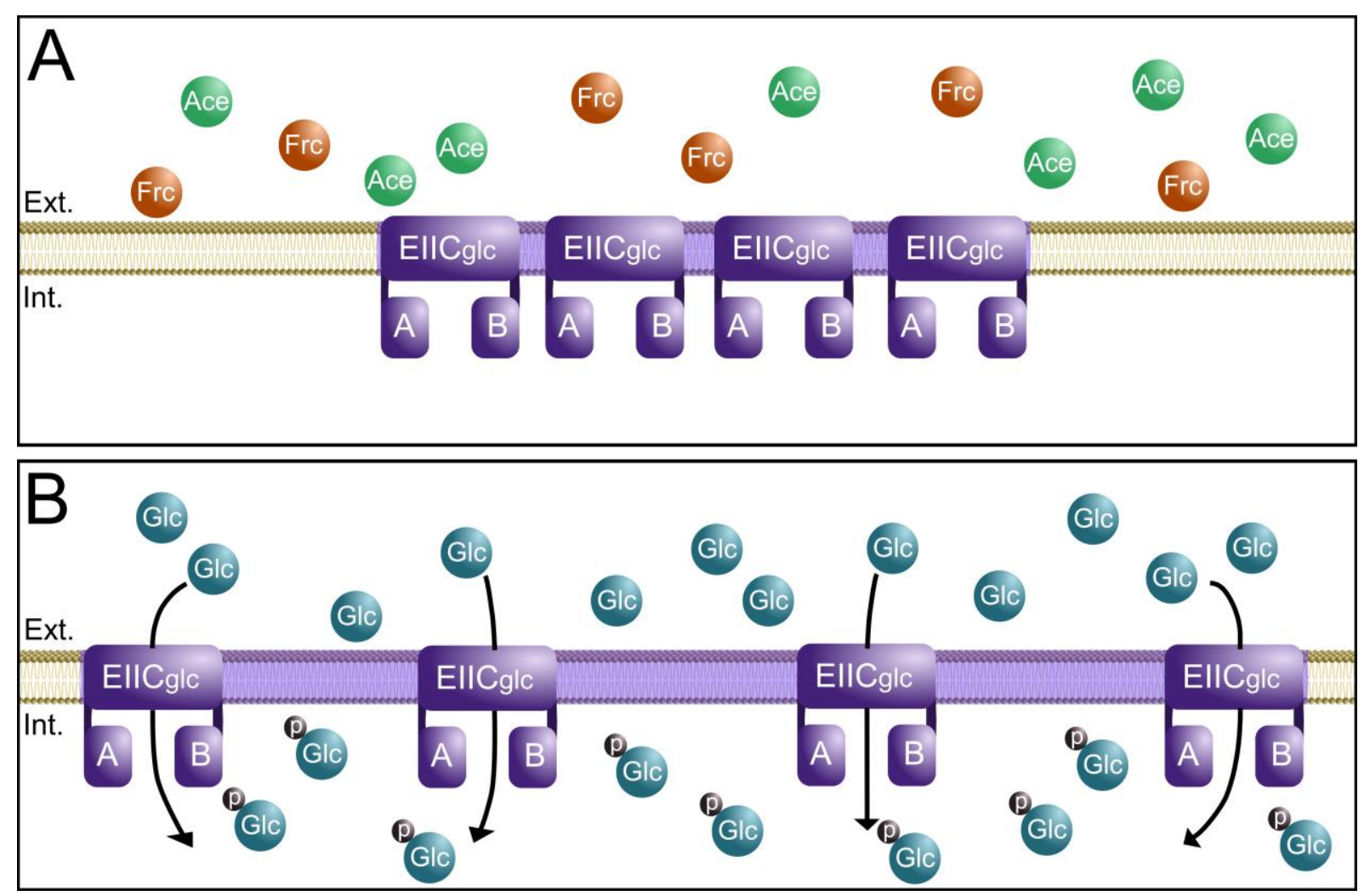

Figure 10. Membrane integral PtsG cluster undergo spatial rearrangement in

971 presence of glucose. Schematic view of PtsG membrane occupancy in $C$.

972 glutamicum. (A) In absence of the PTS sugar, in this case glucose, PtsG cluster are

973 densely packed with proteins. These clusters occupy only a minimal membrane area.

(B) In presence of the correct PTS substrate PtsG clusters rearrange, reducing the overall cluster density and occupying larger membrane areas. 
Keywords: Protein dynamics, PTS, Membrane occupancy, fluorescence microscopy,

Ludwig-Maximilians-Universität München, Fakultät Biologie, Großhaderner Straße 2-

990

4, 82152 Planegg-Martinsried, Germany

991

992

993

994

995

996

997 *Corresponding author: Marc Bramkamp

998 Email: marc.bramkamp@lmu.de; Phone: +49-(0)89-218074611; Fax: +49-(0)89-

$999 \quad 218074621$ 
Table S1. Oligonucleotides used in this study

\begin{tabular}{|c|c|c|}
\hline Oligonucleotide & Sequence & $\begin{array}{l}\text { Restriction } \\
\text { site }\end{array}$ \\
\hline $\begin{array}{l}\text { PtsG upstr fw } \\
\text { HindlII }\end{array}$ & ATTAAGCTTTTTTGGCGGGCG & HindlII \\
\hline PtsG upstr rev Xbal & GGGTCTAGAGTCAAACCTTTCTAAACG & Xbal \\
\hline $\begin{array}{l}\text { PtsG n-ter fwd } \\
\text { Xmal }\end{array}$ & TATACCCGGGATGGCGTCCAAA & Xmal \\
\hline $\begin{array}{l}\text { PtsG n-ter rev } \\
\text { EcoRI }\end{array}$ & AGGGGA ATTCGAGTGCAGGAATACATAAG & EcoRI \\
\hline $\begin{array}{l}\text { PtsF upstr fwd } \\
\text { Hindlll }\end{array}$ & AAAAAGCTTGCTGTCGATACCTCAGAC & HindIII \\
\hline PtsF upstr rev Xbal & $\begin{array}{l}\text { AAAGTTTCTAGAGCTGATTCTTTCAATCCTT } \\
\text { TG }\end{array}$ & Xbal \\
\hline $\begin{array}{l}\text { PtsF N-ter fwd } \\
\text { Xmal }\end{array}$ & $\begin{array}{l}\text { GTACCCGGGATGAATAGCGTAAATAATTCC } \\
\text { TCGC }\end{array}$ & Xmal \\
\hline $\begin{array}{l}\text { PtsF N-ter rev } \\
\text { EcoRI }\end{array}$ & ATATGAATTCGCAACCGCCGCCGC & EcoRI \\
\hline mCherry fwd Xbal & AAATCTAGAATGGTGAGCAAGGGCGAG & Xbal \\
\hline mCherry rev Xmal & TTACCCGGGCTTGTACAGCTC & Xmal \\
\hline mNeonG fwd Xbal & AAATCTAGAATGGTGAGCAAGGGCGAG & Xbal \\
\hline $\begin{array}{l}\text { mNeonG rev Xmal } \\
\text { linker }\end{array}$ & $\begin{array}{l}\text { AAACCCGGGAATTCGCCAGAACCAGCAGC } \\
\text { GGAGCCAGCGCTTGTACAGCTCGTC }\end{array}$ & Xmal \\
\hline $\begin{array}{l}\text { Fw sdhA C-ter fw } \\
\text { HindllI }\end{array}$ & ATTAAGCTTCAGCGATTGCG & HindIII \\
\hline $\begin{array}{l}\text { Rev sdhA C-ter rev } \\
\text { Xbal }\end{array}$ & TAATCTAGACTTGTAGTTCCTTG & Xbal \\
\hline $\begin{array}{l}\text { Fw sdhA down fw } \\
\text { Xmal }\end{array}$ & TTACCCGGGTAATGAAACTTAC & Xmal \\
\hline $\begin{array}{l}\text { Rev sdhA down rev } \\
\text { EcoRI }\end{array}$ & TTAGAATTCAGCAACACATGC & EcoRI \\
\hline
\end{tabular}


Table S2. Strains and plasmids used in this study

\begin{tabular}{lll}
\hline Strain or plasmid & Relevant characteristics & Source \\
\hline E. coli strains & & \\
DH5 a & F- 880lacZM15 (lacZYA-argF)U169 recA1 & Invitrogen \\
& endA1 hsdR17(rk-,mk+) phoA supE44 thi-1 & \\
& gyrA96 relA1 $\lambda$ -
\end{tabular}

\section{C.glutamicum}

\section{strains}

RES 167

CGM001

CGM002

CGM003

CGM004

CGM005

CGM006

\section{Plasmids}

pK19mobsacB

mNeonGreen-ptsG

pK19msB-mCherry-

ptsF

pK19msB-

PAmCherry-ptsF

pK19msB-

PAmCherry-ptsG

pK19msB-sdhA-

mNeonGreen
Restriction-deficient derivative of strain ATCC Tauch et al. 13032

RES167 derivative with ptsF::mCherry-ptsF This work

RES167 derivative with ptsG::mNeonGreen- This work ptsG

RES167 derivative with ptsF::mCherry-ptsF This work and ptsG::mNeonGreen-ptsG

RES167 derivative with ptsF::PAmCherry-ptsF This work RES167 derivative with ptsG::PAmCherry- This work Linker-ptsG

RES167 derivative with $s d h A:: s d h A-$ mNeonGreen

$\mathrm{Kan}^{\mathrm{r}}$; E. coli/C. glutamicum shuttle vector for construction of insertion and deletion mutants in C. glutamicum

This work

This work

This work

This work

This work 

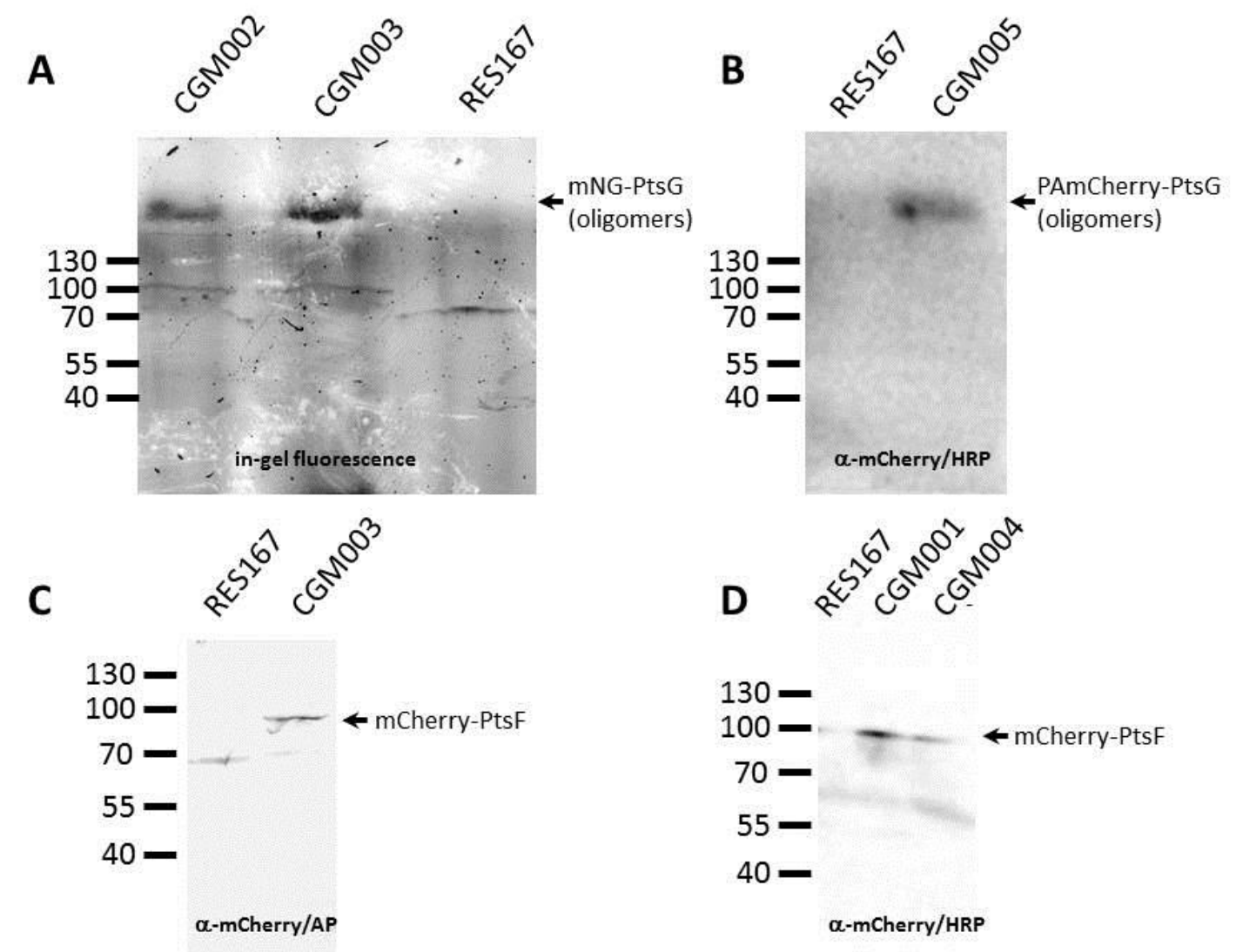

Figure S1. Control for full-length fusion proteins. In gel-fluorescence $(A)$ and western blot analysis (B-D) with cell lysates of strains CGM001-006. (A) In gel fluorescence of cell lysates of CGM002 (mNeonGreen-PtsG) and CGM003 (mNeonGreen-PtsG in double labelled strain background) reveal the existence of oligomeric mNeonGreenPtsG. (B) Western blot of cell lysate of CGM005 (PA-mCherry-PtsG) developed with $\alpha$-mCherry antibodies and HRP-coupled secondary antibodies. PA-mCherry-PtsG is detected as oligomeric band with no apparent degradation products. (C) Western blot of cell lysate of CGM003 (mCherry-PtsF) developed with $\alpha$-mCherry and AP-coupled secondary antibodies. (D) Western blot of cell lysate of CGM001 (mCherry-PtsF) and CGM004 (PA-mCherry-PtsF) developed with $\alpha$-mCherry and HRP-coupled secondary antibodies. Note that little degradation is observed for all fusion constructs. PtsF $=70.51 \mathrm{kDa}, \mathrm{PtsG}=72.57 \mathrm{kDa}$, mCherry $=28.8 \mathrm{kDa}, \mathrm{mNeonGreen}$

kDa. 


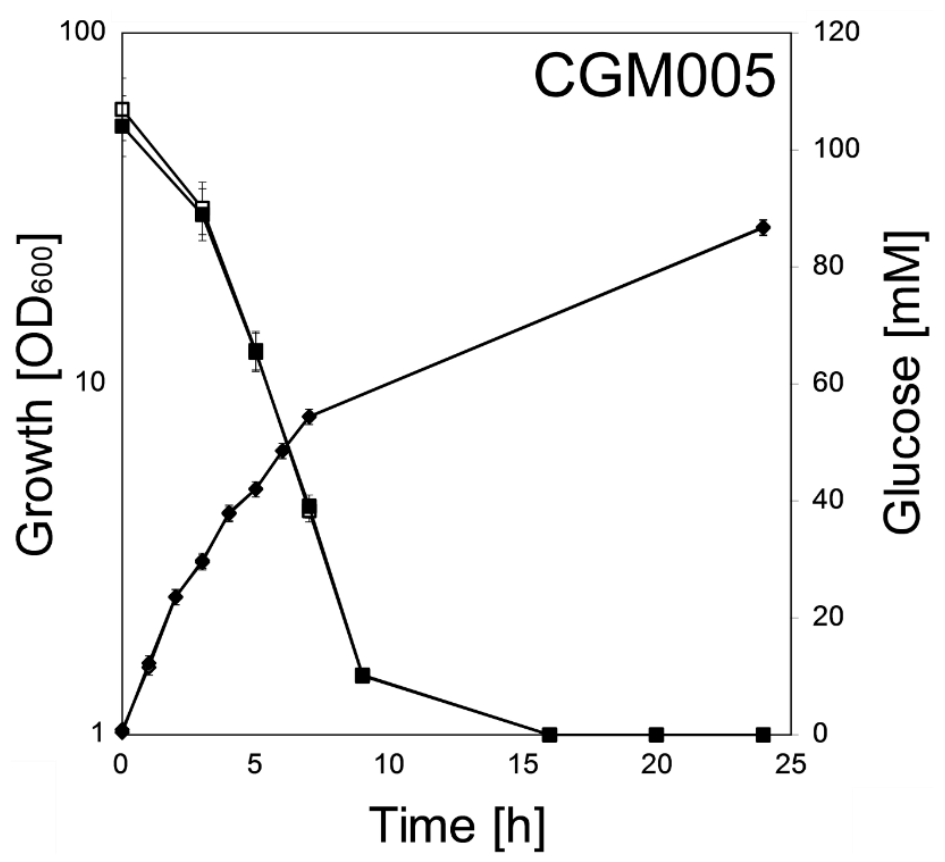

1027 Figure S2. Growth and sugar consumption of C. glutamicum strain CGM005 1028 (PAmCherry-ptsG filled symbols) versus wild type RES 167 (open symbols) on CGXII 1029 containing $100 \mathrm{mM}$ glucose. Glucose consumption (squares) and growth (diamonds) are indicated. Each point represents biological triplicates and standard deviation is indicated. 

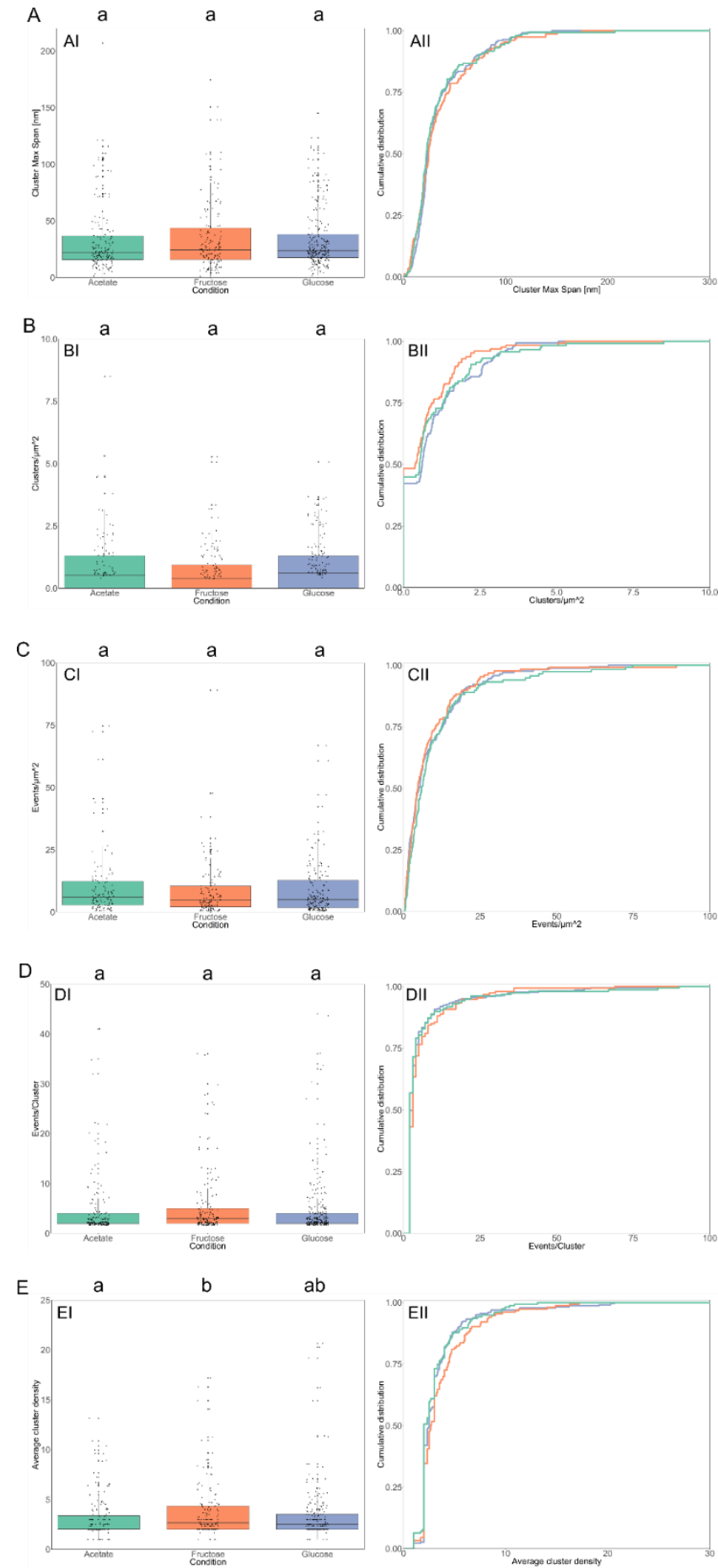

1036 Figure S3. Statistical analysis of single molecule resolution PALM data of $C$. 1037 glutamicum strain ptsF::PAmCherry-ptsF under different growth conditions. (A) PAmCherry-PtsF clusters Maximum Span, (B) PAmCherry-PtsF clusters per $\mu \mathrm{m}^{2},(\mathrm{C})$ 

average density of clusters composed of $>2$ molecules: the arithmetic average of

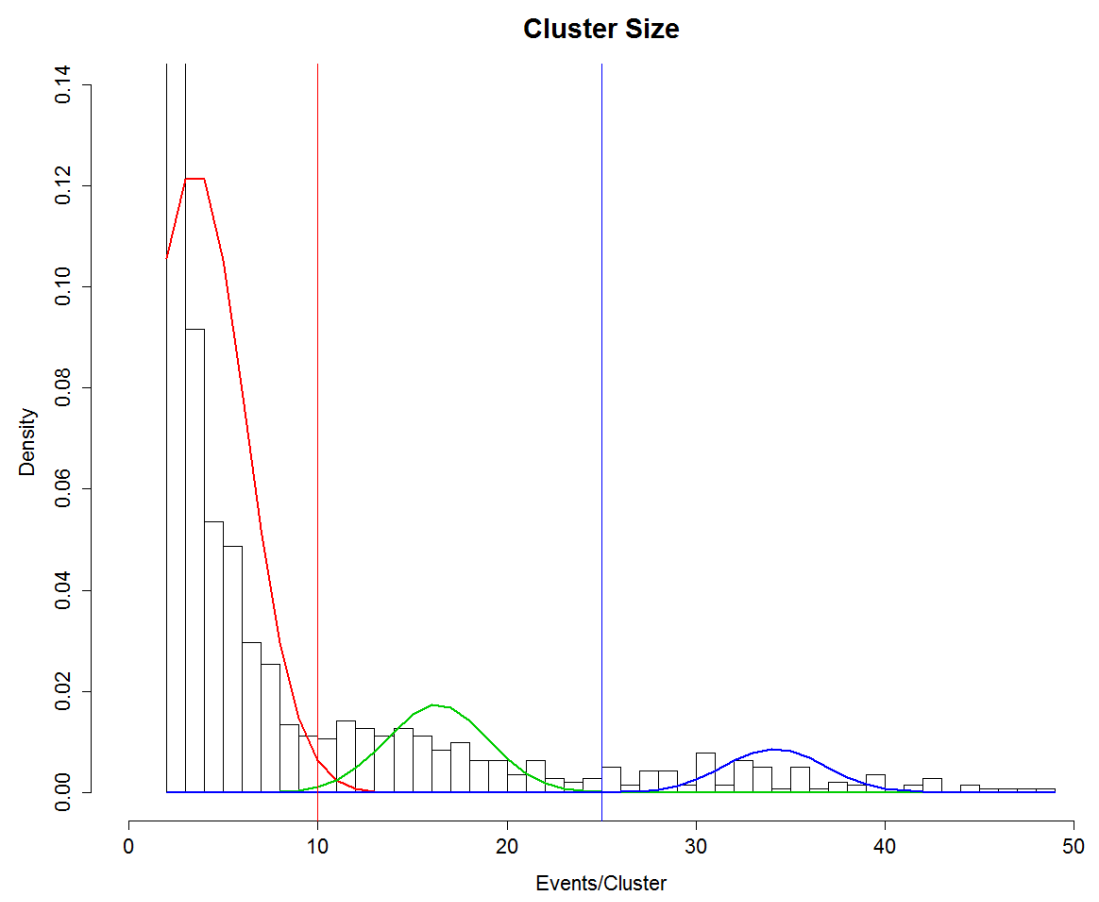

1046 Figure S4. PtsG-PAmCherry clusters size distribution. Three different populations were identified by univariate normal mixture analysis: clusters composed of 2-9, 1024, >25 events. Lines of different colors represent Gaussian fits for each different population. 